\title{
Thermodynamics of magnetized binary compact objects
}

\author{
Kōji Uryū, ${ }^{1}$ Eric Gourgoulhon, ${ }^{2}$ and Charalampos Markakis ${ }^{3}$ \\ 1 Department of Physics, University of the Ryukyus, Senbaru, Nishihara, Okinawa 903-0213, Japan \\ ${ }^{2}$ Laboratoire Univers et Théories, UMR 8102 du CNRS, \\ Observatoire de Paris, Université Paris Diderot, F-92190 Meudon, France \\ ${ }^{3}$ Department of Physics, University of Wisconsin-Milwaukee, P.O. Box 413, Milwaukee, WI 53201
}

(Dated: 28 August 2010)

\begin{abstract}
Binary systems of compact objects with electromagnetic field are modeled by helically symmetric Einstein-Maxwell spacetimes with charged and magnetized perfect fluids. Previously derived thermodynamic laws for helically-symmetric perfect-fluid spacetimes are extended to include the electromagnetic fields, and electric currents and charges; the first law is written as a relation between the change in the asymptotic Noether charge $\delta Q$ and the changes in the area and electric charge of black holes, and in the vorticity, baryon rest mass, entropy, charge and magnetic flux of the magnetized fluid. Using the conservation laws of the circulation of magnetized flow found by Bekenstein and Oron for the ideal magnetohydrodynamic (MHD) fluid, and also for the flow with zero conducting current, we show that, for nearby equilibria that conserve the quantities mentioned above, the relation $\delta Q=0$ is satisfied. We also discuss a formulation for computing numerical solutions of magnetized binary compact objects in equilibrium with emphasis on a first integral of the ideal MHD-Euler equation.
\end{abstract}

PACS numbers:

\section{INTRODUCTION}

Recent observations of anomalous X-ray pulsars, or soft $\gamma$-ray repeaters suggest the existence of neutron stars associated with magnetic fields strong enough to affect their structures in hydrostationary equilibrium (see, e.g. [1]). Such objects have not been found in binary neutron star systems, but hypothetically strongly magnetized neutron stars or black holes may form binary neutron star or black hole - neutron star systems. In this article, we model such magnetized binary compact objects in close circular orbits, assuming that the spacetime and magnetic fields satisfy a helical symmetry and that the stars are in equilibrium.

The helically symmetric spacetime was introduced by Blackburn and Detweiler [2] to model binary compact objects in close circular orbits in general relativity. In such spacetimes, equal amounts of ingoing and outgoing radiation are propagating, and hence these spacetimes do not admit flat asymptotics, because the steady radiation field carries an infinite amount of energy. Nevertheless, it is expected that such a spacetime has an approximate asymptotic region up to a certain radius, where gravitational waves are propagating in a curved background, and the energy of radiation does not dominate in the gravitational mass of the system. Such a solution, however, has not yet been calculated successfully in the regime of strong gravity. Analogously to Schild's result in electromagnetism for two oppositely charged point particles [4], circular orbits of two point particles have been obtained in post-Minkowskian spacetimes [3]. More studies for the helically symmetric spacetimes have been reported by several authors $5-14$.

In 7] (hereafter FUS), thermodynamic laws for helically symmetric perfect fluid spacetimes have been de- rived. In the first part of this paper, we extend the results of FUS to the magnetized perfect-fluid Einstein-Maxwell spacetimes with helical symmetry. As in FUS, we use a helical Killing vector $k^{\alpha}$ to define a conserved Noether current and associated Noether charge $Q$ [15 21]. With an appropriate choice of the current and a constant of the electric potential, the charge $Q$ becomes finite and is independent of the 2-surface $S$ on which it is evaluated as long as the matter and black holes are enclosed in $S$. We obtain the first law, which relates the change $\delta Q$ to the changes in the baryon mass, entropy, circulation and electric current of the fluid, and in the area and electric charge of the black holes. Its expression corresponds to the mass variation formula for stationary axisymmetric spacetimes derived by Carter [22, 23] (Eq. (37) below ${ }^{1}$ ). Concrete calculations for the variation, $\delta Q$, associated with the classical action for an Einstein-Maxwell theory coupled with a perfect fluid carrying an electric current,

$$
\mathcal{L}=\left(\frac{1}{16 \pi} R-\epsilon-\frac{1}{16 \pi} F_{\alpha \beta} F^{\alpha \beta}+A_{\alpha} j^{\alpha}\right) \sqrt{-g},
$$

are summarized in Appendices $\mathrm{A}$ and $\mathrm{B}$ to clarify notation and conventions.

When the late stages of binary inspiral are modeled using a sequence of equilibrium solutions of helically symmetric perfect fluid spacetimes (without electromagnetic fields), the baryon mass, entropy, and circulation of the flow, and the area of each black hole are assumed to be held constant (see e.g. [8, 24 27]). Then, the expression of the first law becomes $\delta Q=0$, or for asymptotically flat systems such as the post-Newtonian, or the

\footnotetext{
${ }^{1}$ The first law Eq. (37) is for generic flows that respect the helical symmetry.
} 
spatially conformally flat systems, $\delta M=\Omega \delta J$, as a result of the conservations of those quantities (FUS). When electromagnetic fields and electric currents are present in neutron stars, the circulation of magnetized flow is not conserved in general. Hence, it is not possible to find a sequence of solutions along which the first law is simplified as above without further assumptions for the flow. In other words, in order to approximate binary inspiral just before a merger by a sequence of quasi-equilibrium solutions, one needs to introduce a model for the evolution of neutron star spins. However, as shown in Sec. III. with an electric current introduced by Bekenstein and Oron for a class of ideal magnetohydrodynamic (MHD) flows (28 30], see also 31] for non-relativistic magnetized flow), a generalized circulation of magnetized flow is found to be conserved. Applying this law - the generalized Kelvin theorem for ideal MHD - we show that the relation $\delta Q=0$ is satisfied along a sequence of helically symmetric equilibria of magnetized binary systems, and that the relation $\delta M=\Omega \delta J$ holds for asymptotically flat systems.

The above first law can be applied to actual sequences of solutions, and hence in the second part of the paper, in Sec. IV and V formulations for computing such equilibrium solutions of magnetized binary compact objects are discussed. In particular, we discuss the first integral of the MHD-Euler equation, which is a key to compute equilibria of neutron stars numerically. Bekenstein and Oron [29] have found a first integral of the relativistic MHDEuler equation using the same current for the case with ideal MHD irrotational flow, and also for the case with the purely convection current. As irrotational flow is considered to be more realistic in the final inspiral stage of the binary neutron stars and the black hole - neutron star binaries [32], we introduce the first integral by Bekenstein and Oron for ideal MHD irrotational flow, then derive a somewhat different first integral, which may be valid only on an initial hypersurface $\Sigma_{t}$, and write down a set of equations for the magnetized irrotational flow suitable for numerical computations of binary neutron stars and black hole-neutron star binaries in equilibrium.

We follow the conventions and notation in FUS. For a one-form $w_{\alpha}$, the exterior derivative $(d w)_{\alpha \beta}$ (within index notation) is defined by

$$
(d w)_{\alpha \beta}:=\nabla_{\alpha} w_{\beta}-\nabla_{\beta} w_{\alpha},
$$

and for a two-form $w_{\alpha \beta}=w_{[\alpha \beta]}$ by

$$
(d w)_{\alpha \beta \gamma}:=3 \nabla_{[\alpha} w_{\beta \gamma]}=\nabla_{\alpha} w_{\beta \gamma}+\nabla_{\beta} w_{\gamma \alpha}+\nabla_{\gamma} w_{\alpha \beta}
$$

\section{THERMODYNAMIC LAWS FOR EINSTEIN-MAXWELL SPACETIME WITH CHARGED AND MAGNETIZED PERFECT FLUID}

\section{A. Zeroth law and constancy of the electric potential on the Killing horizon}

We consider a globally hyperbolic spacetime $\left(\mathcal{M}, g_{\alpha \beta}\right)$ and a vector field $k^{\alpha}$ transverse to each Cauchy surface (but not necessarily everywhere timelike). This vector generates the one-parameter family of diffeomorphisms $\chi_{t}$. The action of $\chi_{t}$ to a spacelike sphere $\mathcal{S}$ on a Cauchy surface generates a timelike surface, $\mathcal{T}(\mathcal{S})=\cup_{t} \chi_{t}(\mathcal{S})$, called the history of $\mathcal{S}$. Then, as in FUS, $k^{\alpha}$ is called a helical vector if there is a smallest $T>0$ for which $P$ and $\chi_{T}(P)$ are timelike separated for every point $P$ outside of the history $\mathcal{T}(\mathcal{S})$. Very often, $k^{\alpha}$ can be written $k^{\alpha}=t^{\alpha}+\Omega \phi^{\alpha}$, where $\Omega=2 \pi / T, t^{\alpha}$ is a timelike vector and $\phi^{\alpha}$ a spacelike vector that has circular orbits with a parameter length $2 \pi$ (see, FUS).

Each Cauchy surface of the helically symmetric spacetime does not admit flat asymptotics because the energy of the radiation generated by a binary equilibrium eventually dominates and causes a divergence. Therefore, as discussed in FUS, the future (past) horizon $\mathcal{H}^{ \pm}$in helically symmetric spacetime is defined by the boundary of the future (past) domain of outer communication $\mathcal{D}^{ \pm}$, where $P \in \mathcal{M}$ is in $\mathcal{D}^{ \pm}$if the future (past) timelike curve $c(\lambda)$ through $P(:=c(0))$ remains outside of $\mathcal{T}(\mathcal{S})$ of each sphere $\mathcal{S}$ for a certain $\lambda_{0}, \lambda>\lambda_{0}$. It is also shown that, if the history $\mathcal{T}(\mathcal{S})$ of a sphere $\mathcal{S}$ is in $\mathcal{D}^{ \pm}$, the future (past) horizon agrees with the chronological past (future) of the history $\mathcal{T}, \mathcal{H}^{ \pm}=\partial I^{\mp}(\mathcal{T})$.

The conditions of the theorems by Friedrich, Rácz, and Wald [33] are modified to make them suitable for helically symmetric spacetimes. With the assumption that the null energy condition holds: $R_{\alpha \beta} l^{\alpha} l^{\beta} \geq 0$ for any null vector $l^{\alpha}$, those theorems yield the following properties: $\mathcal{H}^{ \pm}$are Killing horizons, the shear $\sigma_{\alpha \beta}$ and the expansion $\theta$ of a null congruence vanish on $\mathcal{H}^{ \pm}$, the Killing vector $k^{\alpha}$ is parallel to the null generators of the horizons, and the surface gravity $\kappa$ of each disconnected horizon defined by

$$
k^{\beta} \nabla_{\beta} k^{\alpha}=\kappa k^{\alpha}
$$

is constant on each connected component of $\mathcal{H}^{ \pm}$(FUS).

The Raychaudhuri equation,

$$
\frac{d \theta}{d \lambda}=-R_{\alpha \beta} l^{\alpha} l^{\beta}-2 \sigma_{\alpha \beta} \sigma^{\alpha \beta}-\frac{1}{2} \theta^{2},
$$

is used to demonstrate the above properties. It implies $R_{\alpha \beta} l^{\alpha} l^{\beta}=0$ on the Killing horizons $\mathcal{H}^{ \pm}$. Assuming there exists no material flow through the horizon but there exists an electromagnetic field $F_{\alpha \beta}:=(d A)_{\alpha \beta}=\nabla_{\alpha} A_{\beta}-$ $\nabla_{\beta} A_{\alpha}$, where $A_{\alpha}$ is the electromagnetic potential one- 
form, we have

$$
\begin{aligned}
R_{\alpha \beta} k^{\alpha} k^{\beta} & =8 \pi T_{\alpha \beta}^{\mathrm{F}} k^{\alpha} k^{\beta}=2 F_{\alpha \gamma} F_{\beta}{ }^{\gamma} k^{\alpha} k^{\beta} \\
& =\frac{1}{2}\left(E_{\alpha} E^{\alpha}+B_{\alpha} B^{\alpha}\right)=0
\end{aligned}
$$

on $\mathcal{H}^{ \pm}$, where $T_{\alpha \beta}^{\mathrm{F}}$ is the stress-energy tensor for the electromagnetic field, and $E_{\alpha}$ and $B_{\alpha}$ are the electric and magnetic components with respect to the helical vector defined by ${ }^{2}$

$$
E_{\alpha}:=F_{\alpha \beta} k^{\beta}, \quad B_{\alpha}:=\frac{1}{2} \epsilon_{\alpha \beta \gamma \delta} F^{\beta \gamma} k^{\delta} .
$$

Note that, as a consequence of (6), $E^{\alpha}$ and $B^{\alpha}$ are both null on $\mathcal{H}^{ \pm}$. Using the Cartan identity,

$$
k^{\beta}(d A)_{\beta \alpha}=£_{k} A_{\alpha}-\nabla_{\alpha}\left(k^{\beta} A_{\beta}\right),
$$

and assuming that $A_{\alpha}$ respects the symmetry $£_{k} A_{\alpha}=0$, one can introduce an electric potential in the rotating frame $E_{\alpha}=-\nabla_{\alpha} \Phi^{\mathrm{E}} .{ }^{3}$ Since $E_{\alpha} k^{\alpha}=B_{\alpha} k^{\alpha}=0$ and $E_{\alpha}$ and $B_{\alpha}$ are both null on $\mathcal{H}^{ \pm}, E_{\alpha}$ and $B_{\alpha}$ are necessarily parallel to the null generator on $\mathcal{H}^{ \pm}$. Then, for any vector $\eta^{\alpha}$ tangent to $\mathcal{H}^{ \pm}, \eta^{\alpha} E_{\alpha}=-\eta^{\alpha} \nabla_{\alpha} \Phi^{\mathrm{E}}=0$, which implies that $\Phi^{\mathrm{E}}$ is constant on the Killing horizon $\mathcal{H}^{ \pm}$[22, 23].

The potential $\Phi^{\mathrm{E}}$ is defined globally if the domain of outer communications is simply connected, and $\Phi^{\mathrm{E}}$ is unique up to the constant of integration. The constant may be chosen $\Phi^{\mathrm{E}} \rightarrow 0$ as $r \rightarrow \infty$ for asymptotically flat systems. For the helically symmetric system, we set the constant by the condition

$$
\frac{1}{4 \pi} \oint_{S} k^{\gamma} A_{\gamma} F^{\alpha \beta} d S_{\alpha \beta}=0
$$

on the boundary sphere $S$ which encloses all black holes and neutron stars, and on which a family of Noether charges is defined in the next section ${ }^{4}$. The total electric charge of the system is defined by the surface integral over the sphere $S$,

$$
Q^{\mathrm{E}}:=\frac{1}{4 \pi} \oint_{S} F^{\alpha \beta} d S_{\alpha \beta}
$$

\footnotetext{
2 If $k^{\alpha}$ would be normalized by $k_{\alpha} k^{\alpha}=-1, E_{\alpha}$ and $B_{\alpha}$ could be interpreted physically as the electric and magnetic fields measured by the observer of 4 -velocity $k^{\alpha}$. Note however that in general $k_{\alpha} k^{\alpha} \neq-1$; even $k_{\alpha} k^{\alpha}=0$ on $\mathcal{H}^{ \pm}$.

3 One can avoid the assumption that the field $A_{\alpha}$ respects the helical symmetry. Eq. (7) implies $(d E)_{\alpha \beta}=-k^{\gamma}(d F)_{\gamma \alpha \beta}-£_{k} F_{\alpha \beta}=$ 0 for $(d F)_{\alpha \beta \gamma}=0$ and the symmetry $£_{k} F_{\alpha \beta}=0$. Hence, from the Poincaré lemma, ${ }^{\exists} \Phi^{\mathrm{E}}$ such that $E_{\alpha}=-\nabla_{\alpha} \Phi^{\mathrm{E}}$ if the domain is connected and simply connected.

4 For an asymptotically flat spacetime, the Noether charge defined on $S$ with the choice of Eq. (9), and then the radius of $S$ taken to be $r \rightarrow \infty$, agrees with a choice $\Phi^{\mathrm{E}} \rightarrow 0$ at $r \rightarrow \infty$ (see, Sec. IIB].
}

and the condition (9) is rewritten for $-k^{\alpha} A_{\alpha}=\Phi^{\mathrm{E}}+C$ with

$$
C=-\frac{1}{4 \pi Q^{\mathrm{E}}} \oint_{S} \Phi^{\mathrm{E}} F^{\alpha \beta} d S_{\alpha \beta}
$$

\section{B. First law for systems with a single Killing vector}

\section{Definition of the Noether charge $Q$}

Given a 1-parameter family of magnetized perfect-fluid Einstein-Maxwell spacetimes specified by

$$
\mathcal{Q}(\lambda):=\left[g_{\alpha \beta}(\lambda), u^{\alpha}(\lambda), \rho(\lambda), s(\lambda), A_{\alpha}(\lambda), j^{\alpha}(\lambda)\right],
$$

a family of Noether charges is defined on any sphere $S$ that encloses all black holes and neutron stars associated with the electric charge and current [16-21] :

$$
Q=\oint_{S} Q^{\alpha \beta} d S_{\alpha \beta}
$$

where

$$
Q^{\alpha \beta}=-\frac{1}{8 \pi} \nabla^{\alpha} k^{\beta}+k^{\alpha} \mathfrak{B}^{\beta}-k^{\beta} \mathfrak{B}^{\alpha},
$$

and $\mathfrak{B}^{\alpha}(\lambda)$ is any family of vector fields that satisfies

$$
\frac{1}{\sqrt{-g}} \frac{d}{d \lambda}\left(\mathfrak{B}^{\alpha} \sqrt{-g}\right)=\Theta^{\alpha},
$$

$\Theta^{\alpha}$ being defined by Eq. A30 in Appendix $\mathrm{A}$ The vector $\mathfrak{B}^{\alpha}(\lambda)$ is written,

$$
\begin{gathered}
\mathfrak{B}^{\alpha}(\lambda)=\left.\frac{1}{16 \pi}\left(g^{\alpha \gamma} g^{\beta \delta}-g^{\alpha \beta} g^{\gamma \delta}\right)\right|_{\lambda=0} \stackrel{\circ}{\nabla}_{\beta} g_{\gamma \delta}(\lambda) \\
+\left.\frac{1}{4 \pi} F^{\beta \alpha}\right|_{\lambda=0}\left[A_{\beta}(\lambda)-b A_{\beta}(0)\right]+\mathrm{O}\left(\lambda^{2}\right)
\end{gathered}
$$

where $\stackrel{\circ}{\nabla}_{\beta}$ is the covariant derivative of the metric $g_{\alpha \beta}(0)$ and $b$ is a fixed parameter.

We choose $\mathfrak{B}^{\alpha}(\lambda)$ to make $Q(\lambda)$ finite; and, as we will see below, $Q(\lambda)$ is independent of the sphere $S$, as long as $S$ encloses the fluid and black holes associated with electric charge and current. We first choose the parameter $b$ in definition (16) to have $Q(0)$ satisfy these properties. Regardless of the choice of $\mathfrak{B}^{\alpha}(0)$, the variation of the Noether charge $\delta Q$ is finite and independent of the sphere $S$. The change in the Noether charge $\delta Q$ results in the first law for the Einstein-Maxwell spacetimes with charged and magnetized perfect fluid and Killing horizons, associated with a single Killing vector to impose the stationarity of the system.

In the calculation of the variation $\delta Q$, the Eulerian change of each quantity in Eq. (12) is defined by $\delta \mathcal{Q}:=$ $\frac{d}{d \lambda} \mathcal{Q}(\lambda)$, and the Lagrangian change at $\lambda=0$ is given by

$$
\Delta \mathcal{Q}=\left(\delta+£_{\xi}\right) \mathcal{Q},
$$

where $\xi^{\alpha}$ is a Lagrangian displacement. The definition of Lagrangian perturbations is given in Appendix A 1. 


\section{Independence of $Q(0)$ on the location of $S$}

When the electromagnetic field satisfies $F_{\alpha \beta} F^{\alpha \beta}=0$ in the region where the sphere $S$ is located, $b=1$ is chosen in Eq. (16) to make $Q(0)$ finite and independent of $S$. In this case, we have $\mathfrak{B}^{\alpha}(0)=0$. When the steady electromagnetic radiation is propagating everywhere in the spacetime, $b=1 / 2$ is chosen. Then, $\mathfrak{B}^{\alpha}(0)$ becomes $\mathfrak{B}^{\alpha}(0)=\left.F^{\beta \alpha}\right|_{\lambda=0} A_{\beta}(0) / 8 \pi$. For the former case, a contribution from the gravitational radiation field to the charge $Q(0)$ is subtracted, and for the latter case, contributions from the gravitational and electromagnetic radiation fields to the charge $Q(0)$ are subtracted; $Q(0)$ is finite and independent of $S$ as long as it contains the fluid and all black holes in both cases.

To prove that the charge $Q=Q(0)$ is independent of the sphere $S$, we write $Q=Q_{K}+Q_{L}$, where $Q_{K}$ is the Komar charge and $Q_{L}$ an additional contribution related to the surface term of the Lagrangian, with

$$
\begin{aligned}
Q_{K} & =-\frac{1}{8 \pi} \oint_{S} \nabla^{\alpha} k^{\beta} d S_{\alpha \beta}, \\
Q_{L} & =\oint_{S}\left(k^{\alpha} \mathfrak{B}^{\beta}-k^{\beta} \mathfrak{B}^{\alpha}\right) d S_{\alpha \beta},
\end{aligned}
$$

and rewrite $Q$ in terms of integrals over a spacelike hypersurface $\Sigma$ transverse to $k^{\alpha}$. The boundary of $\Sigma$,

$$
\partial \Sigma=S \cup_{i} \mathcal{B}_{i}
$$

is the union of the sphere $S$ and black hole boundaries $\mathcal{B}_{i}$, which is the $i$ th connected component of $\Sigma \cap \mathcal{H}^{+}$. Correspondingly, surface integrals over the $i$ th black hole horizon $\mathcal{B}_{i}$ are written,

$$
\begin{aligned}
Q_{K i} & =-\frac{1}{8 \pi} \oint_{\mathcal{B} i} \nabla^{\alpha} k^{\beta} d S_{\alpha \beta}, \\
Q_{L i} & =\oint_{\mathcal{B} i}\left(k^{\alpha} \mathfrak{B}^{\beta}-k^{\beta} \mathfrak{B}^{\alpha}\right) d S_{\alpha \beta} .
\end{aligned}
$$

Then, from the identity

$$
\nabla_{\beta} \nabla^{\alpha} k^{\beta}=R_{\beta}^{\alpha} k^{\beta},
$$

we have

$$
\begin{aligned}
& Q_{K}-\sum_{i} Q_{K i} \\
= & -\frac{1}{8 \pi} \int_{\partial \Sigma} \nabla^{\alpha} k^{\beta} d S_{\alpha \beta}=-\frac{1}{8 \pi} \int_{\Sigma} R^{\alpha}{ }_{\beta} k^{\beta} d S_{\alpha} \\
= & -\frac{1}{8 \pi} \int_{\Sigma} G^{\alpha}{ }_{\beta} k^{\beta} d S_{\alpha}-\frac{1}{16 \pi} \int_{\Sigma} R k^{\alpha} d S_{\alpha},
\end{aligned}
$$

where the integral over the boundary $\partial \Sigma$ is related to the surface integrals with the orientations, $\int_{\partial \Sigma} Q^{\alpha \beta} d S_{\alpha \beta}=$ $\left(\oint_{S}-\sum_{i} \oint_{\mathcal{B} i}\right) Q^{\alpha \beta} d S_{\alpha \beta}$. If $F_{\alpha \beta} F^{\alpha \beta}=0$ is satisfied in the neighborhood and outside of the sphere $S$, the vacuum Einstein equation is satisfied in the same region. From
Eq. (24) and the choice $\mathfrak{B}^{\alpha}(0)=0, Q$ is then independent of the location of $S$. For the case $F_{\alpha \beta} F^{\alpha \beta} \neq 0$, using

$$
\begin{aligned}
& Q_{L}-\sum_{i} Q_{L i} \\
& =\int_{\Sigma} \nabla_{\beta}\left(k^{\alpha} \mathfrak{B}^{\beta}-k^{\beta} \mathfrak{B}^{\alpha}\right) d S_{\alpha}=\int_{\Sigma} \nabla_{\beta} \mathfrak{B}^{\beta} k^{\alpha} d S_{\alpha} \\
& =\int_{\Sigma}\left(\frac{1}{8 \pi} \nabla_{\beta} F^{\alpha \beta} A_{\alpha}-\frac{1}{16 \pi} F^{\alpha \beta} F_{\alpha \beta}\right) k^{\gamma} d S_{\gamma}
\end{aligned}
$$

we have

$$
\begin{aligned}
Q & -\sum_{i} Q_{i} \\
= & -\frac{1}{8 \pi} \int_{\Sigma}\left(G^{\alpha}{ }_{\beta}-8 \pi T_{\mathrm{F} \beta}^{\alpha}\right) k^{\beta} d S_{\alpha}-\frac{1}{16 \pi} \int_{\Sigma} R k^{\alpha} d S_{\alpha} . \\
& +\int_{\Sigma}\left(\frac{1}{8 \pi} \nabla_{\gamma} F^{\beta \gamma} A_{\beta} k^{\alpha}-\frac{1}{4 \pi} k^{\gamma} A_{\gamma} \nabla_{\beta} F^{\alpha \beta}\right) d S_{\alpha} \\
& -\sum_{i} \frac{1}{4 \pi} \oint_{\mathcal{B} i} k^{\gamma} A_{\gamma} F^{\alpha \beta} d S_{\alpha \beta} .
\end{aligned}
$$

where $T_{\mathrm{F}}^{\alpha}$, the stress-energy tensor of the electromagnetic field, is defined by Eq. (A6). To derive Eq. (26), we have used the Cartan identity (8), the symmetry relation $£_{k} A_{\alpha}=0$, and Eq. (9). From Eq. (26), it is obvious that $Q$ does not depend on the sphere $S$ as long as it encloses all black holes and neutron stars; all integrands of the volume integrals over $\Sigma$ in Eq. (26) are zero in the region where there are no matters and currents, where the sphere $S$ is placed. This argument may be clearer by using an expression of the Komar charge associated with the Lagrangian, Eq. (B8), given in Appendix B.

\section{First law for the charge $Q$}

The generalized first law will be obtained by evaluating the variation $\delta Q$ in the Noether charge in terms of perturbations of the baryon mass, entropy, circulation and electric current of each fluid element, and the surface areas and charges of the black holes. To find the change $\delta Q$, we first compute the difference,

$$
\delta\left(Q-\sum_{i} Q_{i}\right),
$$

between the charge on the sphere $S$ and the sum of the charges on the black holes $\mathcal{B}_{i}$. The calculation is performed in Appendix B and results in Eq. (B15). In computing the difference (27), we choose two kinds of gauge: the first one is to choose $\delta k^{\alpha}=0$ using the diffeomorphism gauge freedom, and the second one $\xi^{t}=0$ for the Lagrangian displacement as a result of the trivial displacement (see Appendix $\mathrm{B}$ and FUS). For a perfect fluid spacetime, it has been shown in FUS that the quantity (27) is invariant under gauge transformations that respect the Killing symmetry. For the case with an 
electromagnetic field, the same invariance under gauge transformations associated with diffeomorphisms and the $U(1)$ gauge symmetry is shown to hold for the charge $Q$ with a contribution from the electromagnetic fields, as is discussed below.

In the black-hole charges $Q_{i}=Q_{K i}+Q_{L i}, Q_{K i}$ is calculated in FUS:

$$
Q_{K i}=-\frac{1}{8 \pi} \oint_{\mathcal{B} i} \nabla^{\alpha} k^{\beta} d S_{\alpha \beta}=\frac{1}{8 \pi} \kappa_{i} \mathcal{A}_{i},
$$

where $\mathcal{A}_{i}$ is the area of the $i$ th black hole. The $Q_{L i}$ is made of contributions from the geometry, electric charge, and electromagnetic field. The former has been evaluated in FUS following 34]:

$$
\begin{aligned}
\delta Q_{L i} & =\oint_{\mathcal{B} i}\left(k^{\alpha} \Theta^{\beta}-k^{\beta} \Theta^{\alpha}\right) d S_{\alpha \beta} \\
& =-\frac{1}{8 \pi} \delta \kappa_{i} \mathcal{A}_{i}+\frac{1}{4 \pi} \oint_{\mathcal{B} i} k_{\alpha} F^{\beta \alpha} \delta A_{\beta} d \mathcal{A} .
\end{aligned}
$$

For the latter contribution, since $k^{\alpha} F_{\beta \alpha}=E_{\beta}$ is parallel to the null generator $k_{\beta}$ on $\mathcal{H}^{+}$, we have

$$
\begin{aligned}
k_{\alpha} F^{\beta \alpha} \delta A_{\beta} d \mathcal{A} & =k^{\alpha} F_{\beta \alpha} g^{\beta \gamma} \delta A_{\gamma} d \mathcal{A} \\
& =k^{\alpha} F_{\beta \alpha}\left(-k^{\beta} \mathfrak{n}^{\gamma}-\mathfrak{n}^{\beta} k^{\gamma}\right) \delta A_{\gamma} d \mathcal{A} \\
& =k^{\alpha} \mathfrak{n}^{\beta} F_{\alpha \beta} \delta\left(k^{\gamma} A_{\gamma}\right) d \mathcal{A} \\
& =\delta\left(k^{\gamma} A_{\gamma}\right) F^{\alpha \beta} \frac{1}{2}\left(k_{\alpha} \mathfrak{n}_{\beta}-k_{\beta} \mathfrak{n}_{\alpha}\right) d \mathcal{A} \\
& =\delta\left(k^{\gamma} A_{\gamma}\right) F^{\alpha \beta} d S_{\alpha \beta}
\end{aligned}
$$

where $\mathfrak{n}^{\alpha}$ is the unique null vector field orthogonal to $\mathcal{B}_{i}$ satisfying $\mathfrak{n}_{\alpha} k^{\alpha}=-1$, and $\delta k^{\gamma}=0$ is used. Hence

$$
\delta Q_{L i}=-\frac{1}{8 \pi} \delta \kappa_{i} \mathcal{A}_{i}+\frac{1}{4 \pi} \oint_{\mathcal{B} i} \delta\left(k^{\gamma} A_{\gamma}\right) F^{\alpha \beta} d S_{\alpha \beta}
$$

The contributions from the horizon are Eqs. (28) and (31), and the surface integral in the r.h.s. of Eq. (B15),

$$
-\sum_{i} \frac{1}{4 \pi} \delta \oint_{\mathcal{B} i} k^{\gamma} A_{\gamma} F^{\alpha \beta} d S_{\alpha \beta}
$$

Hence the sum of Eqs. (31), (32) and the perturbed (28) becomes

$$
\begin{aligned}
& \delta Q_{i}-\frac{1}{4 \pi} \delta \oint_{\mathcal{B} i} k^{\gamma} A_{\gamma} F^{\alpha \beta} d S_{\alpha \beta}, \\
= & \frac{1}{8 \pi} \kappa_{i} \delta \mathcal{A}_{i}-\frac{1}{4 \pi} \oint_{\mathcal{B} i} k^{\gamma} A_{\gamma} \delta\left(F^{\alpha \beta} d S_{\alpha \beta}\right) \\
= & \frac{1}{8 \pi} \kappa_{i} \delta \mathcal{A}_{i}+\Phi_{i}^{\mathrm{E}} \delta Q_{i}^{\mathrm{E}}
\end{aligned}
$$

where the total electric charge of the system Eq. (10) is rewritten using Stokes' theorem:

$$
Q^{\mathrm{E}}=\int_{\Sigma} j^{\alpha} d S_{\alpha}+\sum_{i} \frac{1}{4 \pi} \oint_{\mathcal{B} i} F^{\alpha \beta} d S_{\alpha \beta},
$$

and the electric charge on each black hole is defined by

$$
Q_{i}^{\mathrm{E}}:=\frac{1}{4 \pi} \oint_{\mathcal{B} i} F^{\alpha \beta} d S_{\alpha \beta}
$$

Note that $\Phi_{i}^{\mathrm{E}}$ is defined on each $\mathcal{B}_{i}$ by

$$
\Phi_{i}^{\mathrm{E}}=-A^{\alpha} k_{\alpha}=\Phi^{\mathrm{E}}+C
$$

and is constant.

Finally, when Einstein's equation, Maxwell's equations, their linear perturbations and the equation of motion are all satisfied, the first law, which relates the change of the Noether charge to changes in the thermodynamic and hydrodynamic equilibrium of matter, in the electric current and electromagnetic field, and in the area and electric charge of the horizon, is derived from Eqs. (B15) and (33),

$$
\begin{aligned}
\delta Q= & \int_{\Sigma}\left\{\frac{T}{u^{t}} \Delta\left(s \rho u^{\alpha} d S_{\alpha}\right)+\frac{h-T s}{u^{t}} \Delta\left(\rho u^{\alpha} d S_{\alpha}\right)\right. \\
& +v^{\beta} \Delta\left(h u_{\beta} \rho u^{\alpha} d S_{\alpha}\right)-A_{\beta} k^{\beta} \Delta\left(j^{\alpha} d S_{\alpha}\right) \\
& \left.-\left(j^{\alpha} k^{\beta}-j^{\beta} k^{\alpha}\right) \Delta A_{\beta} d S_{\alpha}\right\} \\
+ & \sum_{i}\left(\frac{1}{8 \pi} \kappa_{i} \delta \mathcal{A}_{i}+\Phi_{i}^{\mathrm{E}} \delta Q_{i}^{\mathrm{E}}\right) .
\end{aligned}
$$

Here $T$ is the temperature, $s$ the entropy per baryon, $h$ the relativistic enthalpy and $v^{\alpha}$ is defined by the following decomposition of the fluid 4 -velocity with respect to the helical vector:

$$
u^{\alpha}=u^{t}\left(k^{\alpha}+v^{\alpha}\right) \quad \text { with } \quad v^{\alpha} \nabla_{\alpha} t=0 .
$$

Note that $u^{t}=u^{\alpha} \nabla_{\alpha} t$. In the special case of stationary and axisymmetric spacetimes (for which $k^{\alpha}$ is a linear combination of the stationary Killing vector and the axisymmetric one), Eq. (37) reduces to the mass variation formula derived by Carter [22, 23]. ${ }^{5}$

As mentioned earlier, we can verify now that $Q(\lambda)$ is independent of the location of the 2 -surface $S$ on which it is evaluated. In Sec. II B 2, the charge $Q(\lambda)$ at $\lambda=0$ is shown to be independent of $S$, and the variation formula Eq. (37) imply that $d Q / d \lambda=\delta Q$ is independent of $S$ as long as it encloses the fluid and black holes.

\footnotetext{
5 To derive the mass variation formula for stationary and axisymmetric spacetimes from Eq. [37), one can replace the helical vector $k^{\alpha}$ by the timelike killing vector $t^{\alpha}$. All calculations above are valid with this replacement, and now $\delta Q$ becomes $\delta M$ as the sphere $S$ goes to infinity (see Sec. IIB5 and FUS). Extra terms relating to the angular momentum of the fluid and black hole (geometry and electromagnetic field) appear in the r.h.s of Eq. 37] as a result of this replacement.
} 


\section{Gauge invariance of $\delta Q$}

As shown in FUS, for perfect-fluid spacetimes, the difference $\delta\left(Q-\sum_{i} Q_{i}\right)$ is invariant under any gauge transformation associated with diffeomorphisms that respects the symmetry $k^{\alpha}$; in fact $\delta\left(Q_{K}-\sum_{i} Q_{K i}\right)$ and $\delta\left(Q_{L}-\sum_{i} Q_{L i}\right)$ are separately invariant [7]. Because of the contribution from the electric potential at the horizon, $\delta\left(Q-\sum_{i} Q_{i}\right)$ is no longer invariant when an electromagnetic field is present, neither is each contribution. We find, however, that an expression in which the contribution of the electric charge times the potential at the boundary is subtracted,

$$
\delta\left(Q-\sum_{i} Q_{i}-\frac{1}{4 \pi} \int_{\partial \Sigma} k^{\gamma} A_{\gamma} F^{\alpha \beta} d S_{\alpha \beta}\right)
$$

is invariant under the gauge transformation that respects the symmetry and the $U(1)$ gauge transformation as shown below.

The gauge transformation associated with a vector field $\eta^{\alpha}$ is given by

$$
\delta_{\eta} \mathcal{Q}=£_{\eta} \mathcal{Q}, \quad \xi^{\alpha}(\eta)=-\eta^{\alpha},
$$

and the corresponding Lagrangian variation is identically zero,

$$
\Delta_{\eta}=\delta_{\eta}+£_{-\eta}=0 .
$$

We decompose the vector $\eta^{\alpha}$ with respect to the symme$\operatorname{try} k^{\alpha}$,

$$
\eta^{\alpha}=\eta^{\alpha} \nabla_{\alpha} t k^{\alpha}+\hat{\eta}^{\alpha}
$$

with $\hat{\eta}^{\alpha} \nabla_{\alpha} t=0$.

Then, the change in $\delta\left(Q_{L}-\sum_{i} Q_{L i}\right)$ becomes

$$
\begin{gathered}
\delta_{\eta}\left(Q_{L}-\sum_{i} Q_{L i}\right)=\int_{\Sigma} \nabla_{\beta} \Theta^{\beta} k^{\alpha} d S_{\alpha}=\int_{\Sigma} \delta_{\eta} \mathcal{L} d^{3} x \\
=\int_{\Sigma} \nabla_{\alpha}\left(\mathcal{L} \hat{\eta}^{\alpha}\right) d^{3} x=-\frac{1}{8 \pi} \int_{\partial \Sigma} F_{\gamma \delta} F^{\gamma \delta} k^{\alpha} \hat{\eta}^{\beta} d S_{\alpha \beta},
\end{gathered}
$$

where we used the relation $\delta_{\eta} \mathcal{L}=£_{\eta} \mathcal{L}=\nabla_{\alpha}\left(\mathcal{L} \hat{\eta}^{\alpha}\right)=$ $\nabla_{\beta}\left(\mathcal{L} k^{\alpha} \nabla_{\alpha} t \hat{\eta}^{\beta}\right)$, with $k^{\alpha} \nabla_{\alpha} t=1$. The non-zero contribution to the Lagrangian density $\mathcal{L}$ at the boundary $\partial \Sigma$ is that of the electromagnetic field $\mathcal{L}_{\mathrm{F}}$.

Similarly $\delta\left(Q_{K}-\sum_{i} Q_{K i}\right)$ is calculated from Eq. (24):

$$
\begin{aligned}
& \delta_{\eta}\left(Q_{K}-\sum_{i} Q_{K i}\right)=-\frac{1}{8 \pi} \delta_{\eta} \int_{\Sigma} R_{\beta}^{\alpha} k^{\beta} d S_{\alpha} \\
& =-\frac{1}{8 \pi} \int_{\partial \Sigma} 2 R^{\alpha}{ }_{\gamma} k^{\gamma} \hat{\eta}^{\beta} d S_{\alpha \beta} \\
& =-\frac{1}{2 \pi} \int_{\partial \Sigma}\left[£_{k} A_{\delta}-\nabla_{\delta}\left(k^{\gamma} A_{\gamma}\right)\right] F^{\alpha \delta} \hat{\eta}^{\beta} d S_{\alpha \beta} \\
& \quad+\frac{1}{8 \pi} \int_{\partial \Sigma} F_{\gamma \delta} F^{\gamma \delta} k^{\alpha} \hat{\eta}^{\beta} d S_{\alpha \beta},
\end{aligned}
$$

where we have substituted $R_{\beta}^{\alpha}=8 \pi T_{\mathrm{F}}^{\alpha}{ }_{\beta}$ at $\partial \Sigma$ and Eq. (A6), before using Eq. (8). Finally, the last term in Eq. (39) becomes

$$
\begin{aligned}
& -\frac{1}{4 \pi} \delta_{\eta} \int_{\partial \Sigma} k^{\gamma} A_{\gamma} F^{\alpha \beta} d S_{\alpha \beta} \\
= & -\frac{1}{4 \pi} \delta_{\eta} \int_{\Sigma} \nabla_{\delta}\left(k^{\gamma} A_{\gamma} F^{\alpha \delta}\right) d S_{\alpha} \\
= & -\frac{1}{2 \pi} \int_{\partial \Sigma} \nabla_{\delta}\left(k^{\gamma} A_{\gamma} F^{\alpha \delta}\right) \hat{\eta}^{\beta} d S_{\alpha \beta} .
\end{aligned}
$$

Adding Eqs. (44), (43), and (45), and imposing $£_{k} A_{\alpha}=0$ and $\nabla_{\beta} F^{\alpha \beta}=0$ at $\partial \Sigma$, all terms cancel out :

$$
\delta_{\eta}\left(Q-\sum_{i} Q_{i}-\frac{1}{4 \pi} \int_{\partial \Sigma} k^{\gamma} A_{\gamma} F^{\alpha \beta} d S_{\alpha \beta}\right)=0 .
$$

Hence the difference (39) is invariant under a gauge transformation that respects the symmetry.

For the $U(1)$ gauge transformation, we directly show, instead of Eq. (39), the invariance of the difference evaluated at the surface $S$,

$$
\delta\left(Q-\frac{1}{4 \pi} \oint_{S} k^{\gamma} A_{\gamma} F^{\alpha \beta} d S_{\alpha \beta}\right)
$$

under the transformation with a gauge potential $f$,

$$
\delta_{f} A_{\alpha}=\nabla_{\alpha} f
$$

The change in charge $Q$ with this transformation is

$$
\delta_{f} Q=\delta_{f} Q_{L}=\frac{1}{4 \pi} \oint_{S}\left(k^{\alpha} F^{\gamma \beta}-k^{\beta} F^{\gamma \alpha}\right) \delta_{f} A_{\gamma} d S_{\alpha \beta}
$$

Then, the difference (47) vanishes

$$
\begin{aligned}
& \delta_{f}\left(Q-\frac{1}{4 \pi} \int_{S} k^{\gamma} A_{\gamma} F^{\alpha \beta} d S_{\alpha \beta}\right) \\
& =-\frac{3}{4 \pi} \oint_{S} k^{[\alpha} F^{\beta \gamma]} \nabla_{\gamma} f d S_{\alpha \beta}=0
\end{aligned}
$$

because integration by part of the r.h.s. of the first equality becomes an itegration of a divergence over $S$ that vanishes, and an integral of

$$
\begin{aligned}
3 \nabla_{\gamma}\left(k^{[\alpha} F^{\beta \gamma]}\right) & =2 k^{[\alpha} \nabla_{\gamma} F^{\beta] \gamma}+\nabla_{\gamma} k^{\gamma} F^{\alpha \beta}+£_{k} F^{\alpha \beta} \\
& =0
\end{aligned}
$$

when the Maxwell's equation is satisfied on $S$ where the current is zero, and $k^{\alpha}$ the Killing vector.

\section{Asymptotically flat systems}

FUS have derived the first law in a Hamiltonian framework, and shown the relations between $Q_{K}$ and $\delta Q_{L}$ and the asymptotic quantities, the ADM mass $M$, the Komar mass $M_{\mathrm{K}}$ associated with the timelike asymptotic Killing 
vector $t^{\alpha}$, and the angular momentum $J$ associated with the asymptotic rotational Killing vector $\phi^{\alpha}$. In the presence of an electromagnetic field, the only difference with FUS is the following term in $\delta Q_{L}$

$$
\oint_{\infty}\left(k^{\alpha} \Theta_{\mathrm{F}}^{\beta}-k^{\beta} \Theta_{\mathrm{F}}^{\alpha}\right) d S_{\alpha \beta},
$$

where

$$
\oint_{\infty}:=\lim _{r \rightarrow \infty} \oint_{S_{r}},
$$

with $S_{r}$ is a sphere of a radius $r$, and $\Theta_{\mathrm{F}}^{\alpha}$ is the surface term of the variation of electromagnetic Lagrangian defined by

$$
\Theta_{\mathrm{F}}^{\alpha}=\frac{1}{4 \pi} F^{\beta \alpha} \delta A_{\beta}
$$

However, this does not contribute to $\delta Q_{L}$, because, for asymptotically flat systems, the components of $A_{\alpha}$ are $O\left(r^{-1}\right)$ or lower, and, accordingly, those of $F^{\alpha \beta}$ are $O\left(r^{-2}\right)$ or lower, hence

$$
\oint_{\infty}\left(k^{\alpha} \Theta_{\mathrm{F}}^{\beta}-k^{\beta} \Theta_{\mathrm{F}}^{\alpha}\right) d S_{\alpha \beta}=\lim _{r \rightarrow \infty} \oint_{S_{r}} \Theta_{\mathrm{F}}^{\alpha} \nabla_{\alpha} r r^{2} d \Omega=0
$$

where the relations $k^{\alpha} \nabla_{\alpha} t=1$ and $k^{\alpha} \nabla_{\alpha} r=0$ have been used. Therefore, as in FUS,

$$
\begin{aligned}
Q_{K} & =-\frac{1}{8 \pi} \oint_{\infty} \nabla^{\alpha} k^{\beta} d S_{\alpha \beta}=\frac{1}{2} M_{K}-\Omega J \\
\delta Q_{L} & =\oint_{\infty}\left(k^{\alpha} \Theta^{\beta}-k^{\beta} \Theta^{\alpha}\right) d S_{\alpha \beta} \\
& =\delta M-\frac{1}{2} \delta M_{K}+\delta \Omega J
\end{aligned}
$$

which results in

$$
\delta Q=\delta M-\Omega \delta J .
$$

As we will see below, when two nearby equilibria are compared conserving the integral quantities, including the generalized Kelvin circulation for magnetized flow, and the areas and electric charges of the black holes, so that the r.h.s. of Eq. (37) vanishes, the first law is simply written $\delta Q=0$, or $\delta M=\Omega \delta J$ for asymptotically flat systems.

\section{COMPARING STATIONARY SYSTEMS}

\section{A. Ideal MHD flow}

\section{Conservation of rest mass, entropy and electric charge}

For an isentropic fluid, conservation of rest mass and entropy can be expressed by means of a Lie derivative along the fluid 4-velocity $u^{\alpha}$ :

$$
£_{u}(\rho \sqrt{-g})=0, \quad £_{u} s=0
$$

and if these quantities are conserved in the perturbed states, the perturbed conservation laws have first integrals

$$
\Delta(\rho \sqrt{-g})=0, \quad \Delta s=0,
$$

Since we assume that the electric current is not necessarily colinear to the fluid 4-velocity, conservation of electric current,

$$
£_{j} \sqrt{-g}=0,
$$

does not imply another perturbed conservation law analogous to Eq. (60) with respect to the lagrange perturbation of the congruence of flow lines, that is, $\Delta\left(j^{\alpha} d S_{\alpha}\right) \neq$ 0 . However, its volume integral over the neutron star should vanish because of the conservation of electric charge:

$$
\delta Q_{\mathrm{m}}^{\mathrm{E}}=\delta \int_{\Sigma} j^{\alpha} d S_{\alpha}=\int_{\Sigma} \Delta\left(j^{\alpha} d S_{\alpha}\right)=0 .
$$

\section{Conservation of magnetic flux for ideal $M H D$}

Assuming perfect conductivity for the magnetized flow of the neutron star matter, the ideal MHD condition

$$
F_{\alpha \beta} u^{\beta}=0 \text {, }
$$

is satisfied, and the curl of Eq. (63) becomes

$$
£_{u} F_{\alpha \beta}=0
$$

as a result of the Cartan identity and $(d F)_{\alpha \beta \gamma}=0$. Eq. (64) implies the well known conservation law of magnetic flux, Alfven's theorem. Let us introduce the map $\psi_{\tau}$ as the family of diffeomorphisms generated by $u^{\alpha}$, namely the curve $\tau \rightarrow \psi_{\tau}(P)$ has the tangent vector $u^{\alpha}(P)$ at a point $P$. For any closed curve $c$ contractable to a point, we consider the closed curve $c_{\tau}=\psi_{\tau} \circ c$ obtained by moving each point of $c$ during the proper time $\tau$ along the fluid trajectory through that point. Then the conservation of magnetic flux, which is the integral form of the law (64), amounts to the conservation of the integral of the 1-form $A_{\alpha}$ along the closed curve $c_{\tau}$ in the fluid :

$$
\oint_{c_{\tau}} A_{\alpha} d \ell^{\alpha}=C_{\mathrm{F}}=\text { const. }
$$

When the perturbed state also satisfies the ideal MHD condition, the perturbed version of the conservation of magnetic flux (12) has a first integral

$$
\Delta F_{\alpha \beta}=0
$$

and hence $\Delta F_{\alpha \beta}=(d \Delta A)_{\alpha \beta}=0$ is satisfied for any region in the fluid. For $(d \Delta A)_{\alpha \beta}=0$ to be satisfied, it suffices that $\Delta A_{\alpha}=\nabla_{\alpha} \Psi$ for some scalar field $\Psi$. Conversely, as long as the fluid support (neutron star) is starconvex, the Poincaré lemma guarantees the existence of 
$\Psi$. As a result, the last term of the volume integral of Eq. (37) vanishes:

$$
\begin{aligned}
& \int_{\Sigma}-\left(j^{\alpha} k^{\beta}-j^{\beta} k^{\alpha}\right) \Delta A_{\beta} d S_{\alpha} \\
= & \int_{\partial \Sigma}-\left(j^{\alpha} k^{\beta}-j^{\beta} k^{\alpha}\right) \Psi d S_{\alpha \beta} \\
+ & \int_{\Sigma} \nabla_{\beta}\left(j^{\alpha} k^{\beta}-j^{\beta} k^{\alpha}\right) \Psi d S_{\alpha}=0,
\end{aligned}
$$

because there is no electric current on the boundary surface $\partial \Sigma$, and a relation, $\nabla_{\beta}\left(j^{\alpha} k^{\beta}-j^{\beta} k^{\alpha}\right)=£_{k} j^{\alpha}+$ $j^{\alpha} \nabla_{\beta} k^{\beta}-k^{\alpha} \nabla_{\beta} j^{\beta}=0$, is satisfied for the conserved current $j^{\alpha}$ that respects the symmetry.

\section{Conservation of circulation for ideal MHD: generalized Kelvin's Theorem}

When two equilibria of some ideal MHD flow are compared with the same rest mass, same entropy and same magnetic flux, the perturbed conservation laws (60) and (66), as well as Eq. (67) are satisfied. Then the change in the Noether charge (37) becomes

$$
\begin{aligned}
\delta Q & =\int_{\Sigma}\left[v^{\beta} \Delta\left(h u_{\beta} \rho u^{\alpha} d S_{\alpha}\right)-A_{\beta} k^{\beta} \Delta\left(j^{\alpha} d S_{\alpha}\right)\right] \\
& +\sum_{i}\left(\frac{1}{8 \pi} \kappa_{i} \delta \mathcal{A}_{i}+\Phi_{i}^{\mathrm{E}} \delta Q_{i}^{\mathrm{E}}\right) .
\end{aligned}
$$

For some perfect fluid without magnetic field, the circulation of the flow is conserved. The curl of the relativistic Euler equation $u^{\beta} \hat{\omega}_{\beta \alpha}=0$ is written $£_{u} \hat{\omega}_{\alpha \beta}=0$ where $\hat{\omega}_{\alpha \beta}$ is the relativistic vorticity defined by $\hat{\omega}_{\alpha \beta}=$ $(d(h u))_{\alpha \beta}$ and a corresponding integral law, known as Kelvin's theorem, is the conservation of circulation, the line integral of $h u_{\alpha}$ along an arbitrary closed curve comoving with the fluid. As shown in FUS, the contribution from the circulation to the change in the Noether charge $\delta Q$ is included in the term

$$
\int_{\Sigma} v^{\beta} \Delta\left(h u_{\beta} \rho u^{\alpha} d S_{\alpha}\right)
$$

which vanishes when the circulation is conserved in the perturbed flow, for example, when the irrotational flow, or the corotational flow, is maintained. This can be shown in the same way as eliminating a term (67) using the conservation of magnetic flux.

The integral in Eq. (68), however, does not in general vanish for magnetized flows, or even for ideal MHD flows, because of the lack of a conservation of circulation law in the magnetized case. This can be seen from the relativistic MHD-Euler equation which is not the inner product of the fluid 4-velocity and an exact two form, because of the Lorenz force on the right hand side,

$$
u^{\beta}(d(h u))_{\beta \alpha}=\frac{1}{\rho} F_{\alpha \beta} j^{\beta} .
$$

Nevertheless, Bekenstein and Oron [29] (see also [30]) have found that, if the 4-current takes the form

$$
j^{\alpha}=\nabla_{\beta}\left(\rho u^{\alpha} q^{\beta}-\rho u^{\beta} q^{\alpha}\right),
$$

where $q^{\alpha}$ is an auxiliary vector field, one can obtain a generalized conserved circulation for magnetized flow. This 4-current is derived from the variation of a Lagrangian in which the ideal MHD condition is added as an interaction term $\rho q^{\alpha} F_{\alpha \beta} u^{\beta}$ with the Lagrange multiplier $\rho q^{\alpha}$. The form (71) manifestly satisfies the electric charge conservation: $\nabla_{\alpha} j^{\alpha}=0$. Note that, for a given 4-current $j^{\alpha}$, one has the degree of freedom to change $q^{\alpha}$ according to

$$
q^{\alpha} \mapsto q^{\alpha}+\lambda u^{\alpha}
$$

for a scalar $\lambda$ without affecting the value of $j^{\alpha}$.

Using $\nabla_{\alpha}\left(\rho u^{\alpha}\right)=0$ [cf. Eq. (59)], the 4-current (71) can be rewritten

$$
j^{\alpha}=£_{q}\left(\rho u^{\alpha}\right)+\rho u^{\alpha} \nabla_{\beta} q^{\beta} .
$$

Substituting the form into the Lorenz force, we get

$$
\frac{1}{\rho} F_{\alpha \beta} j^{\beta}=\frac{1}{\rho} F_{\alpha \beta} £_{q}\left(\rho u^{\beta}\right)=(d \eta)_{\alpha \beta} u^{\beta},
$$

where 1 -form $\eta_{\alpha}$ is defined by

$$
\eta_{\alpha}:=F_{\alpha \beta} q^{\beta}
$$

and a relation (C1) from Appendix C, which is implied by the ideal MHD condition (63), is used. Note that, thanks to (63), the 1-form $\eta_{\alpha}$ does not depend on the specific choice of $q^{\alpha}$ within the range allowed by (72). By means of (74), the MHD-Euler equation (70) is simply written,

$$
u^{\beta}(d w)_{\beta \alpha}=0
$$

where $w_{\alpha}$ is the generalized momentum 1-form defined by

$$
w_{\alpha}:=h u_{\alpha}+\eta_{\alpha}
$$

From Eq. (76) one can easily deduce a generalised conservation of circulation law for ideal MHD flows. Indeed, defining the vorticity $\omega_{\alpha \beta}$ of a magnetized flow by

$$
\omega_{\alpha \beta}=\nabla_{\alpha} w_{\beta}-\nabla_{\beta} w_{\alpha}=(d w)_{\alpha \beta},
$$

the Cartan identity, combined with Eq. (76) and the identity $d \omega=d^{2} w=0$, yields

$$
£_{u} \omega_{\alpha \beta}=0 .
$$

By means of the Stokes theorem, this conservation law can be put in the following integral form [using the same notation as in Eq. (65)]:

$$
\oint_{c_{\tau}}\left(h u_{\alpha}+\eta_{\alpha}\right) d \ell^{\alpha}=C_{\mathrm{m}}=\text { const. }
$$


This law, which has been first derived by Bekenstein and Oron [29], constitutes a generalization to ideal MHD of the relativistic Kelvin's circulation theorem (which corresponds to $\eta_{\alpha}=0$, see e.g. [35])

One can repeat the same argument as for the magnetic flux in the previous section. The perturbation of Eq. (799) for the magnetized vorticity has first integral

$$
\Delta \omega_{\alpha \beta}=0 \text {, }
$$

which implies $\Delta \omega_{\alpha \beta}=(d \Delta(h u+\eta))_{\alpha \beta}=0$. The Poincaré lemma guarantees that a function $\Psi$ exists on the starconvex fluid support such that $\Delta\left(h u_{\alpha}+\eta_{\alpha}\right)=\nabla_{\alpha} \Psi$.

It is also suggested from Eq. (176) that an irrotational magnetohydrodynamic flow, $\omega_{\alpha \beta}=0$, is described by a velocity potential $\Phi$ that satisfies

$$
h u_{\alpha}+\eta_{\alpha}=\nabla_{\alpha} \Phi
$$

and, in this case, $\Psi=\Delta \Phi$.

\section{First law for the ideal MHD with Bekenstein - Oron current}

For ideal MHD flow with the Bekenstein-Oron current (71), the first law of the form Eq. (68) is further simplified when comparing two nearby equilibria that conserve the circulation of a magnetized flow, (80). Substituting Eq. (71) to the second term of the integrand of the volume integral in Eq. (68), we have

$$
\begin{aligned}
-A_{\beta} k^{\beta} \Delta\left(j^{\alpha} d S_{\alpha}\right) & \\
& =\left(£_{k} A_{\gamma}-k^{\beta} F_{\beta \gamma}\right) \Delta\left[\left(\rho u^{\alpha} q^{\gamma}-\rho u^{\gamma} q^{\alpha}\right) d S_{\alpha}\right] \\
& -\nabla_{\gamma}\left\{A_{\beta} k^{\beta} \Delta\left[\left(\rho u^{\alpha} q^{\gamma}-\rho u^{\gamma} q^{\alpha}\right) d S_{\alpha}\right]\right\} \\
& =v^{\beta} \Delta\left(\eta_{\beta} \rho u^{\alpha} d S_{\alpha}\right)-v^{\beta} q^{\gamma} \Delta F_{\beta \gamma} \rho u^{\alpha} d S_{\alpha} \\
& -\frac{1}{u^{t}} u^{\beta} F_{\beta \gamma} \Delta\left(\rho u^{\alpha} q^{\gamma} d S_{\alpha}\right)-k^{\beta} F_{\beta \gamma} \Delta\left(\rho u^{\gamma} q^{\alpha} d S_{\alpha}\right) \\
& -\nabla_{\gamma}\left\{A_{\beta} k^{\beta} \Delta\left[\left(\rho u^{\alpha} q^{\gamma}-\rho u^{\gamma} q^{\alpha}\right) d S_{\alpha}\right]\right\}
\end{aligned}
$$

where the relation $\Delta \nabla_{\beta}\left(f^{\alpha \beta} d S_{\alpha}\right)=\nabla_{\beta} \Delta\left(f^{\alpha \beta} d S_{\alpha}\right)$, valid for any antisymmetric tensor $f^{\alpha \beta}$, and the Cartan identity (8) are used, and the symmetry $£_{k} A_{\gamma}=0$ is imposed.

Since the ideal MHD condition (63) is satisfied, terms including $F_{\alpha \beta} u^{\beta}$ are discarded. Also a term involving $F_{\alpha \beta} \Delta u^{\beta}$ is discarded, because $\Delta u^{\beta}$ is colinear to $u^{\beta}$ (see Eq. (A10). Substituting Eq. (83) to Eq. (37), the integral of the last term of Eq. (83) becomes a surface integral on $\partial \Sigma$ which vanishes. Hence, the first law (37) for the Bekenstein-Oron formulation of ideal MHD is written

$$
\begin{aligned}
\delta Q=\int_{\Sigma} & \left\{\frac{T}{u^{t}} \Delta\left(s \rho u^{\alpha} d S_{\alpha}\right)+\frac{h-T s}{u^{t}} \Delta\left(\rho u^{\alpha} d S_{\alpha}\right)\right. \\
& +v^{\beta} \Delta\left[\left(h u_{\beta}+\eta_{\beta}\right) \rho u^{\alpha} d S_{\alpha}\right] \\
& -v^{\beta} q^{\gamma} \Delta F_{\beta \gamma} \rho u^{\alpha} d S_{\alpha} \\
& \left.-\left(j^{\alpha} k^{\beta}-j^{\beta} k^{\alpha}\right) \Delta A_{\beta} d S_{\alpha}\right\} \\
+ & \sum_{i}\left(\frac{1}{8 \pi} \kappa_{i} \delta \mathcal{A}_{i}+\Phi_{i}^{\mathrm{E}} \delta Q_{i}^{\mathrm{E}}\right) .
\end{aligned}
$$

Introducing the following notation

$$
\begin{gathered}
d M_{\mathrm{B}}:=\rho u^{\alpha} d S_{\alpha}, \quad d S:=s d M_{\mathrm{B}} \\
d C_{\alpha}:=\left(h u_{\alpha}+\eta_{\alpha}\right) d M_{\mathrm{B}},
\end{gathered}
$$

we further rewrite Eq. (84) as

$$
\begin{aligned}
\delta Q= & \int_{\Sigma}\left\{\frac{T}{u^{t}} \Delta d S+\frac{h-T s}{u^{t}} \Delta d M_{\mathrm{B}}+v^{\alpha} \Delta d C_{\alpha}\right. \\
& \left.-v^{\beta} q^{\gamma} \Delta F_{\beta \gamma} d M_{\mathrm{B}}-\left(j^{\alpha} k^{\beta}-j^{\beta} k^{\alpha}\right) \Delta A_{\beta} d S_{\alpha}\right\} \\
& +\sum_{i}\left(\frac{1}{8 \pi} \kappa_{i} \delta \mathcal{A}_{i}+\Phi_{i}^{\mathrm{E}} \delta Q_{i}^{\mathrm{E}}\right) .
\end{aligned}
$$

When the circulation of magnetized flow is conserved, there exists a potential $\Psi$ such that $\Delta\left(h u_{\alpha}+\eta_{\alpha}\right)=\nabla_{\alpha} \Psi$. Applying an argument analogous to that for the magnetic flux in Sec. III A 2, a term for the circulation of magnetized flow in the r.h.s. of Eq. (84) vanishes:

$$
\begin{aligned}
& \int_{\Sigma} v^{\beta} \Delta\left(h u_{\beta}+\eta_{\beta}\right) \rho u^{\alpha} d S_{\alpha} \\
= & \int_{\Sigma}\left(\rho u^{\alpha} v^{\beta}-\rho u^{\beta} v^{\alpha}\right) \nabla_{\beta} \Psi d S_{\alpha}=0
\end{aligned}
$$

where $v^{\alpha} d S_{\alpha}=0$ is used in the first equality, and the last equality is proved in the same way as in Eq. (67) because of a relation, $\nabla_{\beta}\left(\rho u^{\alpha} v^{\beta}-\rho u^{\beta} v^{\alpha}\right)=\nabla_{\beta}\left(\rho u^{\beta} k^{\alpha}-\right.$ $\left.\rho u^{\alpha} k^{\beta}\right)=-£_{k}\left(\rho u^{\alpha}\right)-\rho u^{\alpha} \nabla_{\beta} k^{\beta}+k^{\alpha} \nabla_{\beta}\left(\rho u^{\beta}\right)=0$. Therefore, the rest mass, entropy, circulation of magnetized flow and magnetic flux are all conserved in the perturbation of ideal MHD flow with the Bekenstein-Oron current (71), namely, Eqs. (60), (66), and (81) are satisfied, the change in the Noether charge (84) becomes

$$
\delta Q=\sum_{i}\left(\frac{1}{8 \pi} \kappa_{i} \delta \mathcal{A}_{i}+\Phi_{i}^{\mathrm{E}} \delta Q_{i}^{\mathrm{E}}\right) .
$$

\section{B. MHD flow without conduction current}

It is expected that the inner core of the neutron star may be composed of a mixture of superfluid protons and high-energy particles. Such flows are well described by an ideal fluid without conduction current but only convection current:

$$
j^{\alpha}=\rho e u^{\alpha}
$$

where $e$ is the electric charge per baryon mass [28]. Conservation of rest mass, $\nabla_{\alpha}\left(\rho u^{\alpha}\right)=0$, and current, $\nabla_{\alpha} j^{\alpha}=0$, imply that the specific charge $e$ is conserved along fluid flow lines,

$$
£_{u} e=0 \text {. }
$$


Substituting the current (89) into the first law (37), we have

$$
\begin{aligned}
& \delta Q=\int_{\Sigma}\left\{\frac{T}{u^{t}} \Delta\left(s \rho u^{\alpha} d S_{\alpha}\right)+\frac{h-T s}{u^{t}} \Delta\left(\rho u^{\alpha} d S_{\alpha}\right)\right. \\
& \left.+v^{\beta} \Delta\left[\left(h u_{\beta}+e A_{\beta}\right) \rho u^{\alpha} d S_{\alpha}\right]-\frac{A_{\beta} u^{\beta}}{u^{t}} \Delta\left(e \rho u^{\alpha} d S_{\alpha}\right)\right\} \\
& +\sum_{i}\left(\frac{1}{8 \pi} \kappa_{i} \delta \mathcal{A}_{i}+\Phi_{i}^{\mathrm{E}} \delta Q_{i}^{\mathrm{E}}\right)
\end{aligned}
$$

and also into the MHD-Euler equation (70),

$$
u^{\beta}(d(h u+e A))_{\beta \alpha}+A_{\beta} u^{\beta} \nabla_{\alpha} e=0 .
$$

As shown in 28], the circulation of the magnetized flow defined by

$$
\Gamma:=\oint_{c_{\tau}}\left(h u_{\alpha}+e A_{\alpha}\right) d \ell^{\alpha} .
$$

is conserved only when the closed curve $c_{\tau}$ is taken along a curve of constant specific charge $e$. If we further assume that the charge is distributed initially satisfying

$$
e=e\left(A_{\alpha} u^{\alpha}\right)
$$

(or merely $e=$ constant in the simplest case), the curl of Eq. (92) becomes a law of conservation of circulation for magnetized flow,

$$
£_{u}(d(h u+e A))_{\beta \alpha}=0,
$$

and $\Gamma$ is constant for any closed curved $c_{\tau}$ comoving with the flow. Then, with the same argument in Sec. II A 4. when nearby equilibrium solutions having the same value of circulation $\Gamma$ are compared, the perturbed conservation law,

$$
\Delta(d(h u+e A))_{\beta \alpha}=0,
$$

is satisfied. Hence, with Eq. (96), a perturbation of Eq. (90),

$$
\Delta e=0,
$$

and conservation of rest mass and entropy (60), the first law for a flow without conduction current is also written simply as Eq. (88). It should be noted that the condition $e=$ constant may not be too restrictive for an application such as the superfluid proton component in a neutron star interior.

\section{INTEGRABILITY CONDITION FOR THE MHD-EULER EQUATION IN IDEAL MHD}

When the stationarity or helical symmetry is imposed explicitly on the (MHD-)Euler equation, it is no longer an evolution equation. In usual methods 24 26], its numerical solution is calculated using its first integral - a sufficient condition for the stationary or helically symmetric (MHD-)Euler equation being satisfied. Therefore, finding the first integral is a key, and also a restriction, for computing equilibrium solutions considered in Sec. III.

As shown in Sec. IIIA3, when the Bekenstein-Oron 4-current (71) is introduced, the relativistic MHD-Euler equation for ideal MHD flows takes the form (76). If we assume that the generalized momentum (77) of the magnetized flow respects the helical symmetry, $£_{k} w_{\alpha}=0$, then a first integral is immediately derived for corotational and irrotational flows, in a way fully analogous with the non-magnetized case [42] (see also [35]): the Cartan identity $k^{\beta} \omega_{\beta \alpha}=£_{k} w_{\alpha}-\nabla_{\alpha}\left(w_{\beta} k^{\beta}\right)$ reduces to $k^{\beta} \omega_{\beta \alpha}=-\nabla_{\alpha}\left(w_{\beta} k^{\beta}\right)$ and, for an irrotational flow $\left(\omega_{\beta \alpha}=0\right)$, or for a corotational one $\left[u^{\alpha}\right.$ colinear to $k^{\alpha}$ so that (76) implies $k^{\beta} \omega_{\beta \alpha}=0$ ], we get the first integral $w_{\alpha} k^{\alpha}=$ const.

However, it turns out that the assumption $£_{k} w_{\alpha}=0$ is too restrictive when applied to a corotating flow. In view of (77) and (75), it would yield the first integral $w_{\alpha} k^{\alpha}=h u_{\alpha} k^{\alpha}+F_{\alpha \beta} k^{\alpha} q^{\beta}=$ const. Now, the colinearity of $k^{\alpha}$ and $u^{\alpha}$, along with the ideal MHD condition (63), implies $F_{\alpha \beta} k^{\beta}=0$. Hence the first integral would reduce to $h u_{\alpha} k^{\alpha}=$ const, i.e. exactly the same as in the perfect fluid case, without any Lorentz force term.

In Bekenstein and Oron's theory [29, 30], the momentum $w_{\alpha}$ defined by (77) and (75) contains the Lagrange multiplier $q^{\alpha}$. Because $q^{\alpha}$ is not a physical quantity, it does not necessarily obey the helical symmetry. This has been noticed by Bekenstein and Oron, but has not been taken into account when the first integral was derived. In this section, we briefly review the properties of the 4-current by Bekenstein and Oron, then derive integrability conditions for the case when $q^{\alpha}$ does not respect the symmetry.

A first integral for an axisymmetric and rigidly rotating neutron star has been derived by Bonazzola, Gourgoulhon, Salgado, and Marck [37] (hereafter BGSM). In Appendix D it is shown that the Bekenstein and Oron theory can also accommodate the BGSM formulation if a term involving $£_{k} q^{\alpha}$ is kept in the MHD-Euler equation.

\section{A. Bekenstein-Oron 4-current}

From (73), the Bekenstein-Oron 4-current can be expressed as

$$
\begin{aligned}
j^{\alpha} & =\frac{1}{\sqrt{-g}} £_{q}\left(\rho u^{\alpha} \sqrt{-g}\right) \\
& =-\rho £_{u} q^{\alpha}+u^{\alpha} \nabla_{\beta}\left(\rho q^{\beta}\right) .
\end{aligned}
$$

Let us recall that $j^{\alpha}$ is invariant under a change of $q^{\alpha}$ of the form (72). Without loss of generality, a condition such as $q^{\alpha} u_{\alpha}=0$, or $q^{\alpha} \nabla_{\alpha} t=0$, may be imposed, although these are not used below.

The 4-current must obey the helical symmetry, namely 
its Lie derivative along $k^{\alpha}$ must vanish:

$$
£_{k} j^{\alpha}=\nabla_{\beta}\left(\rho u^{\alpha} £_{k} q^{\beta}-\rho u^{\beta} £_{k} q^{\alpha}\right)=0,
$$

where $£_{k} q^{\alpha} \neq 0$. Using (98) and (99), we can write

$$
\begin{aligned}
£_{k} j^{\alpha} & =\frac{1}{\sqrt{-g}} £_{[k, q]}\left(\rho u^{\alpha} \sqrt{-g}\right) \\
& =-\rho £_{u} £_{k} q^{\alpha}+u^{\alpha} \nabla_{\beta}\left(\rho £_{k} q^{\beta}\right)=0,
\end{aligned}
$$

where the commutator notation $[k, q]^{\alpha}=£_{k} q^{\alpha}$ is used. Note the commutation relation $£_{k} £_{u}-£_{u} £_{k}=£_{[k, u]}=$ 0 , for $u^{\alpha}$ respects the symmetry. In the above expressions for $£_{k} j^{\alpha}$, it is noticed that we have again the freedom to add to $£_{k} q^{\alpha}$ a vector proportional to $u^{\alpha}$, as $£_{k} q^{\alpha} \mapsto$ $£_{k} q^{\alpha}+\lambda u^{\alpha}$.

\section{B. Helically symmetric MHD-Euler equation}

We first rewrite the MHD-Euler equation by isolating the Lie derivative along the helical vector $k^{\alpha}$. Using the decomposition (38) of the 4-velocity, the MHD-Euler equation (76) divided by $u^{t}$ is written

$$
\begin{aligned}
\left(k^{\beta}+v^{\beta}\right)(d w)_{\beta \alpha} & =-\nabla_{\alpha}\left(w_{\beta} k^{\beta}\right)+£_{k} w_{\alpha}+v^{\beta}(d w)_{\beta \alpha} \\
& =0 .
\end{aligned}
$$

Since $\eta_{\alpha} u^{\alpha}=F_{\alpha \beta} u^{\alpha} q^{\beta}=0$ for ideal MHD, we have

$$
w_{\alpha} u^{\alpha}=\left(h u_{\alpha}+\eta_{\alpha}\right) u^{\alpha}=-h,
$$

hence

$$
w_{\alpha} k^{\alpha}=w_{\alpha}\left(\frac{u^{\alpha}}{u^{t}}-v^{\alpha}\right)=-\left(\frac{h}{u^{t}}+w_{\alpha} v^{\alpha}\right),
$$

Substituting this relation into (103), we obtain

$$
\nabla_{\alpha}\left(\frac{h}{u^{t}}+w_{\beta} v^{\beta}\right)+£_{k} w_{\alpha}+v^{\beta}(d w)_{\beta \alpha}=0 .
$$

Since both $h u_{\alpha}$ and $F_{\alpha \beta}$ respect the helical symmetry, we have, given the definition (77) of $w_{\alpha}$,

$$
£_{k} w_{\alpha}=£_{k}\left(h u_{\alpha}+F_{\alpha \beta} q^{\beta}\right)=F_{\alpha \beta} £_{k} q^{\beta} .
$$

Hence Eq. (106) becomes

$$
\nabla_{\alpha}\left(\frac{h}{u^{t}}+w_{\beta} v^{\beta}\right)+F_{\alpha \beta} £_{k} q^{\beta}+v^{\beta}(d w)_{\beta \alpha}=0 .
$$

Starting from this form of the MHD-Euler equation, let us discuss two cases: the corotational flow and the irrotational one.

a. Corotational flow: The flow is corotational if the fluid 4-velocity is parallel to the Killing vector: $u^{\alpha}=$ $u^{t} k^{\alpha}$. This amounts to setting $v^{\alpha}=0$ in the decomposition (38) of the 4-velocity. Accordingly, Eq. (108) reduces to

$$
\nabla_{\alpha}\left(\frac{h}{u^{t}}\right)+F_{\alpha \beta} £_{k} q^{\beta}=0 .
$$

Note that, thanks to (99) and the ideal MHD condition (63), we have

$$
F_{\alpha \beta} £_{k} q^{\beta}=-\frac{1}{\rho u^{t}} F_{\alpha \beta} j^{\beta}
$$

in the corotating case.

b. Irrotational flow: In the Bekenstein and Oron ideal MHD theory, the magnetized flow is called irrotational when the vorticity $\omega_{\alpha \beta}=(d w)_{\alpha \beta}$ defined by (78) vanishes identically. The MHD-Euler equation (76) is then always satisfied. Via the Poincaré lemma, a flow is irrotational if, and only if, there exists (locally) a potential $\Phi$ such that $w_{\alpha}=\nabla_{\alpha} \Phi$. Since $w_{\alpha} v^{\alpha}=v^{\alpha} \nabla_{\alpha} \Phi=$ $£_{v} \Phi$, and $v^{\beta}(d w)_{\beta \alpha}=0$, Eq. (108) reduces to

$$
\nabla_{\alpha}\left(\frac{h}{u^{t}}+£_{v} \Phi\right)+F_{\alpha \beta} £_{k} q^{\beta}=0 .
$$

Note that, contrary to the corotating case, the contribution of the Lorentz force is divided into two terms: $F_{\alpha \beta} £_{k} q^{\beta}$ and the term involving the potential $\Phi$.

\section{Integrability conditions}

Under the assumption of helical symmetry without any restriction on the fluid flow, the integrability condition for Eq. (108) is that the last two terms in the left hand side be the gradient of a function $f$,

$$
F_{\alpha \beta} £_{k} q^{\beta}+v^{\beta}(d w)_{\beta \alpha}=\nabla_{\alpha} f .
$$

It may also be possible that each term is separately integrable, that is, with two functions $f$ and $g$, each term is a gradient,

$$
F_{\alpha \beta} £_{k} q^{\beta}=-£_{k} q^{\beta}(d A)_{\beta \alpha}=\nabla_{\alpha} f,
$$

and

$$
v^{\beta}(d w)_{\beta \alpha}=\nabla_{\alpha} g .
$$

Therefore, the problem of finding a current with which the helically reduced MHD-Euler equation has a first integral is replaced by the problem of finding the Lagrange multiplier $q^{\alpha}$ that satisfies the above integrability conditions. As mentioned in [29], however, the vector $q^{\alpha}$ is not a freely specifiable quantity, and hence it is not trivial to find such a $q^{\alpha}$, even for corotating or irrotational flow where the $v^{\beta}(d w)_{\beta \alpha}$ term vanishes and the integrability condition reduces to Eq. (113).

\section{FORMULATIONS FOR MAGNETIZED BINARY NEUTRON STARS IN EQUILIBRIUM}

\section{A. Bekenstein and Oron's first integral for magnetized irrotational flow}

As mentioned earlier, assuming the current is written as in Eq. (71), and the vector $q^{\alpha}$ respects the symmetry, the MHD-Euler equation is integrable for irrotational flow. Since the canonical momentum $w_{\alpha}$ defined 
in Eq. (77) respects the symmetry, $£_{k} w_{\alpha}=0$, and the velocity potential for the magnetized irrotational flow is defined by Eq. (82), the first integral is written $£_{k} \Phi=$ constant (which is equivalent to $w_{\alpha} k^{\alpha}=$ constant), or more explicitly, from Eq. (111),

$$
\frac{h}{u^{t}}+£_{v} \Phi=\mathcal{E},
$$

where $\mathcal{E}$ is a constant. Assuming a one-parameter EOS, we have three solvable equations, the normalization condition for the 4-velocity, the first integral, and the rest mass conservation equation, for the three variables $\left\{h, u^{t}, \Phi\right\}$. The equation for $\Phi$ is derived in Sec. VD.

\section{B. A first integral for initial data of irrotational magnetized binaries}

Since part of our motivation for calculating numerical solutions of compact binary systems is to prepare quasiequilibrium solutions that can be used as initial data sets for binary inspiral simulations, we assume that the multiplier $q^{\alpha}$ can be specified freely on an initial spacelike hypersurface $\Sigma_{t}$. Then, when all fields and matter satisfy helical symmetry, and the vector $£_{k} q^{\alpha}$ is, at least instantaneously, proportional to the helical killing vector, the term $F_{\alpha \beta} £_{k} q^{\beta}$ becomes integrable

$$
£_{k} q^{\alpha}=£_{k} q^{t} k^{\alpha}
$$

and the coefficient $£_{k} q^{t}$ is a function of $A_{\beta} k^{\beta}$. Note that the assumption (116) is valid only for irrotational flow; for corotational flow $F_{\alpha \beta} u^{\beta}=0$ implies $F_{\alpha \beta} £_{k} q^{\beta}=$ $£_{k} q^{t} F_{\alpha \beta} k^{\beta}=0$. From the Cartan identity (8) and $£_{k} A_{\alpha}=0$, and the assumption (116), the term (113) becomes

$$
-£_{k} q^{\beta}(d A)_{\beta \alpha}=£_{k} q^{t} \nabla_{\alpha}\left(A_{\beta} k^{\beta}\right) .
$$

Hence, for irrotational flow, Eq. (111) is rewritten

$$
\nabla_{\alpha}\left(\frac{h}{u^{t}}+£_{v} \Phi\right)+£_{k} q^{t} \nabla_{\alpha}\left(A_{\beta} k^{\beta}\right)=0,
$$

and is integrable if there is a function $f$ such that

$$
£_{k} q^{t}=f\left(A_{\beta} k^{\beta}\right)
$$

so that

$$
\frac{h}{u^{t}}+£_{v} \Phi+\int £_{k} q^{t} d\left(A_{\beta} k^{\beta}\right)=\mathcal{E},
$$

where $\mathcal{E}$ is a constant.

If a data set on an initial hypersurface respects helical symmetry permanently, the current should necessarily be stationary, $£_{k} j^{\alpha}=0$. Substituting Eq. (116) into Eq. (102), we have

$$
£_{k} j^{\alpha}=-\rho k^{\alpha} £_{u} £_{k} q^{t}+\rho u^{\alpha} £_{k}^{2} q^{t}=0,
$$

where we have used the facts that $\rho$, or $u^{\alpha}$ respect the symmetry, and a relation $\nabla_{\alpha} k^{\alpha}=0$. When the integrability condition (119) is satisfied, a coefficient of $u^{\alpha}$ in Eq. 1121) vanishes, $£_{k}^{2} q^{t}=£_{k} f\left(A_{\alpha} k^{\alpha}\right)=0$, and hence a sufficient condition for stationarity of the current $£_{k} j^{\alpha}=0$ is that the coefficients of $k^{\alpha}$ in Eq. (121) vanish,

$$
£_{u} £_{k} q^{t}=£_{u} f\left(A_{\alpha} k^{\alpha}\right)=0 .
$$

This condition is equivalent to the component of the ideal MHD condition along $k^{\alpha}$,

$$
k^{\alpha} F_{\alpha \beta} u^{\beta}=-£_{u}\left(A_{\alpha} k^{\alpha}\right)=0,
$$

and is rewritten, on the fluid support of $\Sigma_{t}$, as

$$
£_{v}\left(A_{\alpha} k^{\alpha}\right)=0,
$$

that is, $A_{\alpha} k^{\alpha}$ is constant along the spatial velocity $v^{\alpha}$ defined by Eq. (38). However, as mentioned above, there is no guarantee that solutions calculated from the $q^{\alpha}$ of Eq. (119) satisfies Eq. (122) or (124).

As we choose $£_{k} q^{\alpha}$ to be parallel to $k^{\alpha}$ in (116), we may further restrict $q^{\alpha}$ so that $q^{\alpha}$ itself is parallel to $k^{\alpha}$,

$$
q^{\alpha}=q^{t} k^{\alpha}
$$

We substitute (125) to the current (73) to derive an explicit form for the current $j^{\alpha}$,

$$
\begin{aligned}
j^{\alpha} & =£_{q^{t} k}\left(\rho u^{\alpha}\right)+\rho u^{\alpha} \nabla_{\beta}\left(q^{t} k^{\beta}\right) \\
& =-\rho k^{\alpha} £_{u} q^{t}+\rho u^{\alpha} £_{k} q^{t} .
\end{aligned}
$$

For example,

$$
£_{k} q^{t}=\text { constant }
$$

satisfies the stationarity of the current (122) and

$$
q^{t}=\left[a t+b \phi+f_{q}\left(x^{A}\right)\right] k^{\alpha}
$$

satisfies Eq. (127), where $f_{q}$ is a function of coordinates $x^{A} A=1,2$ orthogonal to $k^{\alpha}, k^{\alpha} \nabla_{\alpha} x^{A}=0$, and $a, b$ are parameters that satisfy

$$
a+b \Omega=1 .
$$

Remember that $t$ parametrizes the foliation and the symmetry vector is normalized as $k^{\alpha} \nabla_{\alpha} t=1$, and $\phi$ parametrizes circular orbits with parameter length $2 \pi$ and $k^{\alpha} \nabla_{\alpha} \phi=\Omega$.

\section{A model with $q^{\alpha}=\left[a t+b \phi+f_{q}\left(x^{A}\right)\right] \hat{q}^{\alpha}$}

We next consider a more general form of $q^{\alpha}$ where neither $q^{\alpha}$ nor $£_{k} q^{\alpha}$ is proportional to $k^{\alpha}$. Separating the dependence on the coordinate associated with the $k^{\alpha}$, we assume the form of the vector $q^{\alpha}$ to be

$$
q^{\alpha}=\left[a t+b \phi+f_{q}\left(x^{A}\right)\right] \hat{q}^{\alpha},
$$


where $\hat{q}^{\alpha}$ respects the symmetry

$$
£_{k} \hat{q}^{\alpha}=0,
$$

and hence the relation

$$
£_{k} q^{\alpha}=\hat{q}^{\alpha}
$$

holds.

For corotational or irrotational flows, the integrability condition (113) is rewritten with the requirement that there exists a function $f$ such that

$$
F_{\alpha \beta} \hat{q}^{\beta}=-\hat{q}^{\beta}(d A)_{\beta \alpha}=\nabla_{\alpha} f,
$$

or using the Cartan identity,

$$
£_{\hat{q}} A_{\alpha}=\nabla_{\alpha}\left(A_{\beta} \hat{q}^{\beta}-f\right) .
$$

When stationarity is imposed to the current, substituting Eq. (132) to Eq. (100), we have

$$
£_{k} j^{\alpha}=\nabla_{\beta}\left(\rho u^{\alpha} \hat{q}^{\beta}-\rho u^{\beta} \hat{q}^{\alpha}\right)=0 .
$$

Then, from Eq. (130) and Eq. (71), the current becomes

$$
j^{\alpha}=\left(\rho u^{\alpha} \hat{q}^{\beta}-\rho u^{\beta} \hat{q}^{\alpha}\right) \nabla_{\beta}\left[a t+b \phi+f_{q}\left(x^{A}\right)\right] .
$$

c. Corotating flow: This model can be applied to corotating flow, as long as one can find a particular form of $\hat{q}^{\alpha}$ that satisfies Eq. (133) as well as the stationarity and ideal MHD conditions consistently. For corotating flow, $u^{\alpha}=u^{t} k^{\alpha}$, Eq. (136) becomes

$$
j^{\alpha}=\rho u^{t} k^{\alpha} \hat{q}^{\beta} \nabla_{\beta}\left[a t+b \phi+f_{q}\left(x^{A}\right)\right]-\rho u^{t} \hat{q}^{\alpha} .
$$

Assuming $f_{q}\left(x^{A}\right)=0$ and using $a+\Omega b=1$, the combination of $t$ and $\phi$ components $j^{\phi}-\Omega j^{t}$ becomes

$$
\begin{aligned}
j^{\phi}-\Omega j^{t} & =\rho u^{t}\left(k^{\alpha} \hat{q}^{\beta}-k^{\beta} \hat{q}^{\alpha}\right) \nabla_{\alpha} \phi \nabla_{\beta} t \\
& =-\rho u^{t}\left(\hat{q}^{\phi}-\Omega \hat{q}^{t}\right) .
\end{aligned}
$$

As discussed in Appendix $\mathrm{D}$, when the system is stationary and axisymmetric, and if $\hat{q}^{\alpha}$ satisfies

$$
\hat{q}^{\alpha}=f\left(A_{\phi}\right) \phi^{\alpha},
$$

the formulation becomes the same as that of [37] for a magnetized rotating neutron star.

d. A trivial model for the irrotational flow: When $\hat{q}^{\alpha}$ is taken to be parallel to $k^{\alpha}$,

$$
\hat{q}^{\alpha}=\hat{q}^{t} k^{\alpha},
$$

with $£_{k} \hat{q}^{t}=0$, the first integral is derived as in the previous section, if $\hat{q}^{t}$ is a function of $A_{\alpha} k^{\alpha}$; Eq. (133) becomes

$$
-\hat{q}^{\beta}(d A)_{\beta \alpha}=\hat{q}^{t} \nabla_{\alpha}\left(A_{\beta} k^{\beta}\right)=\nabla_{\alpha} f .
$$

The current (136) in this case is written

$$
j^{\alpha}=\rho u^{t}\left\{v^{\alpha}-k^{\alpha} v^{\beta} \nabla_{\beta}\left[b \phi+f_{q}\left(x^{A}\right)\right]\right\} \hat{q}^{t} .
$$

A trivial solution to the condition (141) is

$$
\hat{q}^{t}=\text { constant. }
$$

\section{Equation for the velocity potential $\Phi$}

To write down an equation for the velocity potential $\Phi$ for magnetized irrotational flow used in an actual numerical code, we introduce a $3+1$ decomposition of the spacetime. In this section, spatial indices are Latin. The spacetime $\mathcal{M}=\mathbb{R} \times \Sigma$ is foliated by a family of spacelike hypersurfaces $\left(\Sigma_{t}\right)_{t \in \mathbb{R}}$ parametrized by $t$. The futurepointing unit normal to the hypersurface $\Sigma_{t}$ is defined by $n_{\alpha}=-\alpha \nabla_{\alpha} t$, where $\alpha$ is the lapse function. Then the generator of time translations in an inertial frame $t^{\alpha}$, and rotating frame (helical vector) $k^{\alpha}$ are related to $n^{\alpha}$ by $t^{\alpha}=\alpha n^{\alpha}+\beta^{\alpha}$ and $k^{\alpha}=\alpha n^{\alpha}+\omega^{\alpha}$ respectively, where $\beta^{\alpha}$ and $\omega^{\alpha}$ denote a spatial shift vector in each frame, and are related by $\omega^{\alpha}=\beta^{\alpha}+\Omega \phi^{\alpha}$. The spatial metric $\gamma_{a b}(t)$ induced on $\Sigma_{t}$ by the spacetime metric $g_{\alpha \beta}$ is equal to the projection tensor orthogonal to $n^{\alpha}, \gamma_{\alpha \beta}=g_{\alpha \beta}+n_{\alpha} n_{\beta}$, restricted to $\Sigma_{t}$. In a chart $\left(t, x^{a}\right)$, the metric $g_{\alpha \beta}$ has the form

$$
d s^{2}=-\alpha^{2} d t^{2}+\gamma_{a b}\left(d x^{a}+\beta^{a} d t\right)\left(d x^{b}+\beta^{b} d t\right) .
$$

The covariant derivative associated with the spatial metric $\gamma_{a b}$ is denoted by $D_{a}$.

In the formulation for irrotational flow, the number of independent variables becomes three [25, 42]. As independent variables, we choose the relativistic enthalpy per baryon mass, the time component of the 4-velocity, and the velocity potential, $\left\{h, u^{t}, \Phi\right\}$. For the first two variables, the first integral Eq. (118) and the normalization of the 4 -velocity $u_{\alpha} u^{\alpha}=-1$ are solved. Using a relation derived from Eqs. (38) and (82),

$$
v_{a}+\omega_{a}=\frac{1}{h u^{t}}\left(D_{a} \Phi-\eta_{a}\right),
$$

these equations are rewritten,

$$
\begin{aligned}
& \frac{h}{u^{t}}+v^{a} D_{a} \Phi+\int £_{k} q^{t} d\left(A_{\alpha} k^{\alpha}\right)=\mathcal{E}, \\
& h^{2}\left[\left(\alpha u^{t}\right)^{2}-1\right]=\left(D^{a} \Phi-\eta^{a}\right)\left(D_{a} \Phi-\eta_{a}\right),
\end{aligned}
$$

where $\eta_{a}$ is a spatial projection of $\eta_{\alpha}, \eta_{a}=\gamma_{a}{ }^{\alpha} \eta_{\alpha}$.

An equation to calculate the velocity potential $\Phi$ is derived from the rest mass conservation law, Eq. (59),

$$
\begin{aligned}
\frac{1}{\sqrt{-g}} £_{u}(\rho \sqrt{-g}) & =\frac{1}{\alpha \sqrt{\gamma}} £_{v}\left(\rho u^{t} \alpha \sqrt{\gamma}\right) \\
& =\frac{1}{\alpha} D_{a}\left(\alpha \rho u^{t} v^{a}\right)=0 .
\end{aligned}
$$

Substituting Eq. (145) in the above relation, we have an elliptic equation for $\Phi$,

$D^{a} D_{a} \Phi=D_{a}\left(\eta^{a}+h u^{t} \omega^{a}\right)-\left(D_{a} \Phi-\eta_{a}-h u^{t} \omega_{a}\right) \frac{h}{\alpha \rho} D^{a} \frac{\alpha \rho}{h}$.

This equation is solved with a Neumann boundary condition to impose the fluid 4 -velocity $u^{\alpha}$ to follow the surface 
of the star. The surface is defined by the vanishing pressure $p=0$, where the relativistic enthalpy is chosen to be $h=1$ which is always possible when a one-parameter equation of state is assumed. Hence, the boundary condition is written

$$
u^{\alpha} \nabla_{\alpha} h=0 \quad \text { at } \quad h=1 .
$$

and, using $£_{k} h=0$ and Eq. (145), it is rewritten,

$$
\left(D^{a} \Phi-\eta^{a}-h u^{t} \omega^{a}\right) D_{a} h=0 .
$$

where $\nabla_{\alpha} h$ and $D_{a} h$ are normal to the stellar surface.

\section{DISCUSSION}

\section{A. First law associated with the Bekenstein and Oron Lagrangian}

The Lagrangian density of the Bekenstein and Oron ideal MHD theory [29] is based on Schutz's Lagrangian density for relativistic fluids [43]. Our Lagrangian density for a relativistic fluid $\mathcal{L}_{\mathrm{m}}=-\epsilon \sqrt{-g}$ (A18), and the Lagrangian variation applied to it, is equivalent for the purpose of deriving the first law. Then, we rewrite the Lagrangian corresponding to that of Bekenstein and Oron as

$$
\mathcal{L}=\left(\frac{1}{16 \pi} R-\epsilon-\frac{1}{16 \pi} F_{\alpha \beta} F^{\alpha \beta}+F_{\alpha \beta} \rho u^{\alpha} q^{\beta}\right) \sqrt{-g},
$$

in which the interaction term is replaced by a term $F_{\alpha \beta} u^{\alpha}$ times the Lagrange multiplier $\rho q^{\alpha}$ which enforces the ideal MHD condition $F_{\alpha \beta} u^{\alpha}=0$.

Associating this Lagrangian with the charge $Q$ (13), we can derive the first law; a calculation of the variation $\delta Q$ is shown in Appendix E Now, the derived first law is for the ideal MHD flow, while our first law (37) is valid for more general MHD flows. Obviously, the argument in Sec. III applies to the case with the Lagrangian (152). Hence, if a sequence of magnetized binary solutions in equilibrium is constructed assuming conservation of rest mass, entropy, magnetized circulation, magnetic flux, black hole surface area and charge for a black hole neutron star binary, the first law in the form $\delta Q=0$, or $\delta M=\Omega \delta J$ for asymptotically flat systems, is satisfied as for non-magnetized ones, and for the latter case, one can apply a turning point theorem to locate a point where the stability of solution changes [44].

\section{B. First integral of MHD-Euler equation}

As mentioned in Sec. IV a first integral of the relativistic MHD-Euler equation is almost crucial for developing a successful method to compute equilibrium binary solutions numerically. When we derive a first integral, we need to specify a form of the vector $q^{\alpha}$, which should be consistent with the stationarity as well as the ideal MHD condition. However, since $q^{\alpha}$ is not a freely specifiable vector, it is not guaranteed that a set of equations admit such a $q^{\alpha}$ as solution in general. Also a difficulty to have a helically symmetric irrotational binary solution in ideal MHD may be explained physically as follows. Because the magnetic flux is frozen into the fluid for ideal MHD, when the binary system is seen in the rotating frame, a poloidal component of the magnetic field may be winded up, since the neutron star is spinning in this frame. This argument does not rule out the possibility to have a helically symmetric magnetized binary neutron stars, although it is not trivial at all to find a $q^{\alpha}$ that gives such solutions.

In Sec. V] we discuss a formulation for computing equilibrium solutions of magnetized binary neutron stars and a possible candidate for a first integral of the relativistic MHD-Euler equation in ideal MHD flows. Our proposal is to assume $£_{k} q^{\alpha}$ be proportional to the helical vector $k^{\alpha}$. It could be possible that this condition is violated as the solution is evolved in time, that is, a solution calculated from the first integral in the Appendix might not respect the helical symmetry or the ideal MHD condition. It would be applicable, however, for computing initial data for merger simulations of magnetized compact objects, because it may be allowed to freely specify $£_{k} q^{\alpha}$, at least instantaneously on an initial hypersurface.

In Sec. $\mathrm{V}$ we also write down a set of equations to be solved for an equilibrium of irrotational neutron star in a binary system. The formulation for solving the Einstein and Maxwell equations are not presented in this paper. In usual ideal MHD simulations, the electric current $j^{\alpha}$ does not contain dynamical degrees of freedom and, accordingly, the Maxwell equation becomes an evolution equation for the magnetic flux density. This equation is again hard to integrate when the stationarity condition is imposed. Therefore our plan is to choose the electromagnetic potential one form $A_{\alpha}$ as a variable and to write the Maxwell equation as a set of elliptic equations. These elliptic equations can be solved with the same numerical method we have developed to solve for the metric potentials of gravitational fields [14, 25]. Our next project is to develop such a numerical code.

\section{Acknowledgments}

We thank Brandon Carter, John L. Friedman and Ichiro Oda for enlightening discussions and suggestions. This work was supported by JSPS Grant-in-Aid for Scientific Research(C) 20540275, MEXT Grant-in-Aid for Scientific Research on Innovative Area 20105004, NSF grants No. PHY0071044 and PHY0503366, NASA grant No. NNG05GB99G, and ANR grant 06-2-134423 Méthodes mathématiques pour la relativité générale. CM thanks the Greek State Scholarships Foundation for support during the early stages of this work and the Paris Observatory and the University of Wisconsin-Milwaukee 
for travel support. KU and EG acknowledge support from the JSPS Invitation Fellowship for Research in Japan (Short-term) and the invitation program of foreign researchers at the Paris Observatory.

\section{Appendix A: Variation of the Lagrangian}

We begin with a classical action for an EinsteinMaxwell theory coupled with a perfect fluid carrying electric current,

$$
\begin{gathered}
S=\int \mathcal{L} d^{4} x \\
\mathcal{L}=\mathcal{L}_{\mathrm{G}}+\mathcal{L}_{\mathrm{m}}+\mathcal{L}_{\mathrm{F}}+\mathcal{L}_{\mathrm{I}} \\
=\left(\frac{1}{16 \pi} R-\epsilon-\frac{1}{16 \pi} F_{\alpha \beta} F^{\alpha \beta}+A_{\alpha} j^{\alpha}\right) \sqrt{-g} .
\end{gathered}
$$

We first define the Lagrange perturbation for the fluid.

\section{Lagrange displacement}

We describe a perfect fluid by its four-velocity $u^{\alpha}$ and stress-energy tensor

$$
T^{\alpha \beta}=\epsilon u^{\alpha} u^{\beta}+p q^{\alpha \beta},
$$

where $p$ is the fluid's pressure, $\epsilon$ its energy density, and

$$
q^{\alpha \beta}=g^{\alpha \beta}+u^{\alpha} u^{\beta}
$$

is the projection tensor orthogonal to $u^{\alpha}$. We assume that the fluid satisfies an equation of state of the form

$$
p=p(\rho, s), \epsilon=\epsilon(\rho, s),
$$

with $\rho$ the baryon-mass density and $s$ the entropy per unit baryon mass. (That is, $\rho:=m_{B} n$, with $n$ the number density of baryons and $m_{B}$ the mean baryon mass.)

The electromagnetic stress-energy tensor is given by

$$
T_{\mathrm{F}}^{\alpha \beta}=\frac{1}{4 \pi}\left(F^{\alpha \gamma} F_{\gamma}^{\beta}-\frac{1}{4} g^{\alpha \beta} F_{\gamma \delta} F^{\gamma \delta}\right),
$$

where electromagnetic field 2-form $F_{\alpha \beta}$ relates to the potential 1-form by

$$
F_{\alpha \beta}=(d A)_{\alpha \beta}=\nabla_{\alpha} A_{\beta}-\nabla_{\beta} A_{\alpha} .
$$

Given a family of magnetized perfect-fluid EinsteinMaxwell spacetimes specified by

$$
\mathcal{Q}(\lambda):=\left[g_{\alpha \beta}(\lambda), u^{\alpha}(\lambda), \rho(\lambda), s(\lambda), A_{\alpha}(\lambda), j^{\alpha}(\lambda)\right],
$$

one defines the Eulerian change in each quantity by $\delta \mathcal{Q}:=$ $\frac{d}{d \lambda} \mathcal{Q}(\lambda)$.
We introduce a Lagrangian displacement $\xi^{\alpha}$ in the following way: Let $\mathcal{Q}:=\mathcal{Q}(\lambda)$, and let $\Psi_{\lambda}$ be a diffeomorphism mapping each trajectory (worldline) of the initial fluid to a corresponding trajectory of the configuration $\mathcal{Q}(\lambda)$. Then the tangent $\xi^{\alpha}(P)$ to the path $\lambda \rightarrow \Psi_{\lambda}(P)$ can be regarded as a vector joining the fluid element at $P$ in the configuration $\mathcal{Q}(\lambda)$ to a fluid element in a nearby configuration. The Lagrangian change in a quantity at $\lambda=0$, is then given by

$$
\Delta \mathcal{Q}:=\left.\frac{d}{d \lambda} \Psi_{-\lambda} \mathcal{Q}(\lambda)\right|_{\lambda=0}=\left(\delta+£_{\xi}\right) \mathcal{Q} .
$$

The fact that $\Psi_{\lambda}$ maps fluid trajectories to fluid trajectories and the normalization $u^{\alpha} u_{\alpha}=-1$ imply

$$
\Delta u^{\alpha}=\frac{1}{2} u^{\alpha} u^{\beta} u^{\gamma} \Delta g_{\beta \gamma} .
$$

\section{Variation of Lagrangian}

Although the variation of the Lagrangian density (A2) is well known, those calculations are summarized below to clarify notation and conventions. A surface term $\Theta^{\alpha}$ is kept for the calculation of the first law in Sec. IIB

The variation of the Einstein-Hilbert Lagrangian is written as

$$
\begin{aligned}
& \frac{1}{\sqrt{-g}} \delta \mathcal{L}_{\mathrm{G}}=-\frac{1}{16 \pi} G^{\alpha \beta} \delta g_{\alpha \beta}+\nabla_{\alpha} \Theta_{\mathrm{G}}^{\alpha} \\
& \Theta_{\mathrm{G}}^{\alpha}=\frac{1}{16 \pi}\left(g^{\alpha \gamma} g^{\beta \delta}-g^{\alpha \beta} g^{\gamma \delta}\right) \nabla_{\beta} \delta g_{\gamma \delta} .
\end{aligned}
$$

The variation of the perfect fluid Lagrangian is described by the Lagrange perturbations. Considering general perturbations in which the entropy and baryon mass of each fluid element are not conserved along the family $\mathcal{Q}(\lambda)$, one obtains

$$
\frac{\Delta \rho}{\rho}=-\frac{1}{\rho \sqrt{-g}} u_{\alpha} \Delta\left(\rho u^{\alpha} \sqrt{-g}\right)-\frac{1}{2} q^{\alpha \beta} \Delta g_{\alpha \beta} ;
$$

and the local first law of thermodynamics for the fluid,

$$
\Delta \epsilon=\rho T \Delta s+h \Delta \rho,
$$

with the relativistic enthalpy $h$ defined by

$$
h=\frac{\epsilon+p}{\rho},
$$

yields

$$
\frac{\Delta \epsilon}{\epsilon+p}=\frac{\rho T}{\epsilon+p} \Delta s+\frac{\Delta \rho}{\rho} .
$$

Hence, we have

$$
\Delta \epsilon=\rho T \Delta s-\frac{1}{\sqrt{-g}} h u_{\alpha} \Delta\left(\rho u^{\alpha} \sqrt{-g}\right)-\frac{1}{2}(\epsilon+p) q^{\alpha \beta} \Delta g_{\alpha \beta} .
$$


From these relations, the variation of the Lagrangian density for a perfect fluid

$$
\mathcal{L}_{\mathrm{m}}=-\epsilon \sqrt{-g}
$$

becomes

$$
\begin{aligned}
\frac{1}{\sqrt{-g}} \delta \mathcal{L}_{\mathrm{m}}= & -\frac{1}{\sqrt{-g}} \delta(\epsilon \sqrt{-g}) \\
= & -\frac{1}{\sqrt{-g}} \Delta(\epsilon \sqrt{-g})+\frac{1}{\sqrt{-g}} £_{\xi}(\epsilon \sqrt{-g}) \\
= & -\Delta \epsilon-\epsilon \frac{1}{2} g^{\alpha \beta} \Delta g_{\alpha \beta}+\nabla_{\alpha}\left(\epsilon \xi^{\alpha}\right) \\
= & -\rho T \Delta s+\frac{1}{\sqrt{-g}} h u_{\alpha} \Delta\left(\rho u^{\alpha} \sqrt{-g}\right) \\
& +\frac{1}{2} T^{\alpha \beta} \delta g_{\alpha \beta}-\xi_{\alpha} \nabla_{\beta} T^{\alpha \beta}+\nabla_{\alpha} \Theta_{\mathrm{m}}^{\alpha},
\end{aligned}
$$

with the surface term

$$
\Theta_{\mathrm{m}}^{\alpha}=(\epsilon+p) q^{\alpha \beta} \xi_{\beta} .
$$

The variation of the Lagrangian for the electromagnetic field

$$
\mathcal{L}_{\mathrm{F}}=-\frac{1}{16 \pi} F_{\alpha \beta} F^{\alpha \beta} \sqrt{-g}
$$

is calculated as

$$
\begin{aligned}
\frac{1}{\sqrt{-g}} \delta \mathcal{L}_{\mathrm{F}}= & -\frac{1}{16 \pi \sqrt{-g}} \delta\left(F_{\alpha \beta} F^{\alpha \beta} \sqrt{-g}\right) \\
= & -\frac{1}{16 \pi}\left[2(d \delta A)_{\alpha \beta} F^{\alpha \beta}+2 F_{\alpha \gamma} F_{\beta}^{\gamma} \delta g^{\alpha \beta}\right. \\
& \left.\quad+2 F_{\gamma \delta} F^{\gamma \delta} \frac{1}{2} g^{\alpha \beta} \delta g_{\alpha \beta}\right] \\
= & \frac{1}{2} T_{\mathrm{F}}^{\alpha \beta} \delta g_{\alpha \beta}-\frac{1}{4 \pi} \nabla_{\beta} F^{\alpha \beta} \delta A_{\alpha}+\nabla_{\alpha} \Theta_{\mathrm{F}}^{\alpha}
\end{aligned}
$$

where $\Theta_{\mathrm{F}}^{\alpha}$ is defined by

$$
\Theta_{\mathrm{F}}^{\alpha}=\frac{1}{4 \pi} F^{\beta \alpha} \delta A_{\beta}
$$

The variation of the interaction term between matter and the electromagnetic field,

$$
\mathcal{L}_{\mathrm{I}}=A_{\alpha} j^{\alpha} \sqrt{-g},
$$

becomes

$$
\begin{aligned}
\frac{1}{\sqrt{-g}} \delta \mathcal{L}_{\mathrm{I}}= & \delta A_{\alpha} j^{\alpha}+A_{\alpha} \frac{1}{\sqrt{-g}} \Delta\left(j^{\alpha} \sqrt{-g}\right) \\
& -A_{\alpha} \frac{1}{\sqrt{-g}} £_{\xi}\left(j^{\alpha} \sqrt{-g}\right) .
\end{aligned}
$$

Using the relation

$$
\frac{1}{\sqrt{-g}} £_{\xi}\left(j^{\alpha} \sqrt{-g}\right)=\nabla_{\beta}\left(j^{\alpha} \xi^{\beta}-j^{\beta} \xi^{\alpha}\right)+\xi^{\alpha} \nabla_{\beta} j^{\beta},
$$

we have

$$
\begin{aligned}
\frac{1}{\sqrt{-g}} \delta \mathcal{L}_{\mathrm{I}} & =j^{\alpha} \delta A_{\alpha}+A_{\alpha} \frac{1}{\sqrt{-g}} \Delta\left(j^{\alpha} \sqrt{-g}\right) \\
& +\xi^{\alpha}\left[F_{\alpha \beta} j^{\beta}-A_{\alpha} \nabla_{\beta} j^{\beta}\right]+\nabla_{\alpha} \Theta_{\mathrm{I}}^{\alpha},
\end{aligned}
$$

where the surface term is defined by

$$
\Theta_{\mathrm{I}}^{\alpha}=A_{\beta}\left(j^{\alpha} \xi^{\beta}-j^{\beta} \xi^{\alpha}\right)
$$

Variation of the Lagrangian density: Finally, the above terms are collected and the variation of the Lagrangian density $\mathrm{A2}$ is derived,

$$
\begin{aligned}
\frac{1}{\sqrt{-g}} \delta \mathcal{L}= & \frac{1}{\sqrt{-g}}\left(\delta \mathcal{L}_{\mathrm{G}}+\delta \mathcal{L}_{\mathrm{m}}+\delta \mathcal{L}_{\mathrm{F}}+\delta \mathcal{L}_{\mathrm{I}}\right) \\
= & -\rho T \Delta s+\frac{1}{\sqrt{-g}} h u_{\alpha} \Delta\left(\rho u^{\alpha} \sqrt{-g}\right) \\
& +A_{\alpha} \frac{1}{\sqrt{-g}} \Delta\left(j^{\alpha} \sqrt{-g}\right) \\
& -\frac{1}{16 \pi}\left[G^{\alpha \beta}-8 \pi\left(T^{\alpha \beta}+T_{\mathrm{F}}^{\alpha \beta}\right)\right] \delta g_{\alpha \beta} \\
& -\frac{1}{4 \pi}\left(\nabla_{\beta} F^{\alpha \beta}-4 \pi j^{\alpha}\right) \delta A_{\alpha} \\
& -\xi^{\alpha}\left[\nabla_{\beta} T_{\alpha}^{\beta}-F_{\alpha \beta} j^{\beta}+A_{\alpha} \nabla_{\beta} j^{\beta}\right] \\
& +\nabla_{\alpha} \Theta^{\alpha},
\end{aligned}
$$

where the surface term $\Theta^{\alpha}$ is defined by

$$
\begin{aligned}
\Theta^{\alpha}= & \Theta_{\mathrm{G}}^{\alpha}+\Theta_{\mathrm{m}}^{\alpha}+\Theta_{\mathrm{F}}^{\alpha}+\Theta_{\mathrm{I}}^{\alpha} \\
= & \frac{1}{16 \pi}\left(g^{\alpha \gamma} g^{\beta \delta}-g^{\alpha \beta} g^{\gamma \delta}\right) \nabla_{\beta} \delta g_{\gamma \delta}+\frac{1}{4 \pi} F^{\beta \alpha} \delta A_{\beta} \\
& +(\epsilon+p) q^{\alpha \beta} \xi_{\beta}+A_{\beta}\left(j^{\alpha} \xi^{\beta}-j^{\beta} \xi^{\alpha}\right) .
\end{aligned}
$$

\section{Appendix B: Calculation of $\delta\left(Q-\sum_{i} Q_{i}\right)$}

In calculating a contribution from the volume integral to the charge (27), we restrict the gauge in two ways: We use the diffeomorphism gauge freedom to set $\delta k^{\alpha}=$ 0 . The description of fluid perturbations in terms of a Lagrangian displacement $\xi^{\alpha}$ has a second kind of gauge freedom: a class of trivial displacements, including all displacements of the form $f u^{\alpha}$, yield no Eulerian change in the fluid variables. We use this freedom to set $\Delta t=0$. Because $\delta t=0$ ( $t$ is not dynamical), this is equivalent to the condition $\xi^{t}=0$. The relation A10 now implies

$$
\frac{\Delta u^{t}}{u^{t}}=\frac{1}{2} u^{\alpha} u^{\beta} \Delta g_{\alpha \beta}
$$

Then, from Eqs. (A10) and (B1), we have $\Delta u^{\alpha}=$ $\Delta u^{t}\left(k^{\alpha}+v^{\alpha}\right)$, while, by $u^{\alpha}=u^{t}\left(k^{\alpha}+v^{\alpha}\right), \Delta u^{\alpha}=$ $\Delta\left[u^{t}\left(k^{\alpha}+v^{\alpha}\right)\right] ;$ thus

$$
\Delta\left(k^{\alpha}+v^{\alpha}\right)=0
$$


Then, in the variation of the Lagrangian density (A29), a term involving a perturbation of the rest mass density is rewritten

$$
h u_{\alpha} \Delta\left(\rho u^{\alpha} \sqrt{-g}\right)=-\frac{h}{u^{t}} \Delta\left(\rho u^{t} \sqrt{-g}\right) .
$$

To find the change $\delta Q$ in the Noether charge, we first compute the difference,

$$
\delta\left(Q-\sum_{i} Q_{i}\right)
$$

between the charge on the sphere $S$ and the sum of the charges on the black holes $\mathcal{B}_{i}$.

The difference in the Komar charge Eq. (24) is associated with the Lagrangian density as

$$
\begin{aligned}
& Q_{K}-\sum_{i} Q_{K i} \\
= & -\int_{\Sigma}\left(\frac{1}{16 \pi} R-\epsilon-\frac{1}{16 \pi} F_{\alpha \beta} F^{\alpha \beta}+A_{\alpha} j^{\alpha}\right) k^{\gamma} d S_{\gamma} \\
& -\int_{\Sigma}\left(T^{\alpha}{ }_{\beta}+T_{\mathrm{F} \beta}^{\alpha}\right) k^{\beta} d S_{\alpha} \\
& -\int_{\Sigma}\left(\epsilon+\frac{1}{16 \pi} F_{\alpha \beta} F^{\alpha \beta}-A_{\alpha} j^{\alpha}\right) k^{\gamma} d S_{\gamma} \\
& -\frac{1}{8 \pi} \int_{\Sigma}\left[G^{\alpha}{ }_{\beta}-8 \pi\left(T_{\beta}^{\alpha}+T_{\mathrm{F}}^{\alpha}{ }^{\alpha}\right)\right] k^{\beta} d S_{\alpha} .
\end{aligned}
$$

Using the relations

$$
\begin{aligned}
-T_{\beta}^{\alpha} k^{\beta} d S_{\alpha} & =-T_{\beta}^{\alpha}\left(k^{\beta}+v^{\beta}\right) d S_{\alpha}+T^{\alpha}{ }_{\beta} v^{\beta} d S_{\alpha} \\
& =\epsilon k^{\alpha} d S_{\alpha}+(\epsilon+p) u^{\alpha} u_{\beta} v^{\beta} d S_{\alpha},
\end{aligned}
$$

and

$$
\begin{aligned}
- & T_{\mathrm{F}}^{\alpha} \beta k^{\beta} d S_{\alpha}-\left(\frac{1}{16 \pi} F_{\alpha \beta} F^{\alpha \beta}+A_{\alpha} j^{\alpha}\right) k^{\gamma} d S_{\gamma} \\
= & -\frac{1}{4 \pi} F^{\alpha \gamma}\left[£_{k} A_{\gamma}-\nabla_{\gamma}\left(k^{\beta} A_{\beta}\right)\right] d S_{\alpha}+A_{\alpha} j^{\alpha} k^{\gamma} d S_{\gamma} \\
= & -\frac{1}{4 \pi} F^{\alpha \gamma} £_{k} A_{\gamma} d S_{\alpha}+\frac{1}{4 \pi} \nabla_{\gamma}\left(F^{\alpha \gamma} k^{\beta} A_{\beta}\right) d S_{\alpha} \\
& -\frac{1}{4 \pi} k^{\gamma} A_{\gamma}\left(\nabla_{\beta} F^{\alpha \beta}-4 \pi j^{\alpha}\right) d S_{\alpha} \\
& +A_{\alpha}\left(j^{\alpha} k^{\gamma}-j^{\gamma} k^{\alpha}\right) d S_{\gamma},
\end{aligned}
$$

Eq. (B5) is rewritten

$$
\begin{aligned}
& Q_{K}-\sum_{i} Q_{K i} \\
= & -\int_{\Sigma} \mathcal{L} d^{3} x+\int_{\Sigma}(\epsilon+p) u^{\alpha} u_{\beta} v^{\beta} d S_{\alpha} \\
& -\frac{1}{4 \pi} \int_{\Sigma} F^{\alpha \gamma} £_{k} A_{\gamma} d S_{\alpha}+\frac{1}{4 \pi} \int_{\partial \Sigma} k^{\gamma} A_{\gamma} F^{\alpha \beta} d S_{\alpha \beta} \\
& +\int_{\Sigma} A_{\beta}\left(j^{\beta} k^{\alpha}-j^{\alpha} k^{\beta}\right) d S_{\alpha} \\
& -\frac{1}{8 \pi} \int_{\Sigma}\left[G^{\alpha}{ }_{\beta}-8 \pi\left(T^{\alpha}{ }_{\beta}+T_{\mathrm{F}}^{\alpha}\right)\right] k^{\beta} d S_{\alpha} \\
& -\frac{1}{4 \pi} \int_{\Sigma} k^{\gamma} A_{\gamma}\left(\nabla_{\beta} F^{\alpha \beta}-4 \pi j^{\alpha}\right) d S_{\alpha} .
\end{aligned}
$$

The variation of Eq. (B8) is then

$$
\begin{aligned}
& \delta\left(Q_{K}-\sum_{i} Q_{K i}\right) \\
= & -\int_{\Sigma} \delta \mathcal{L} d^{3} x+\int_{\Sigma} \Delta\left[(\epsilon+p) u^{\alpha} u_{\beta} v^{\beta} d S_{\alpha}\right] \\
& -\frac{1}{4 \pi} \delta \int_{\Sigma} F^{\alpha \gamma} £_{k} A_{\gamma} d S_{\alpha}+\frac{1}{4 \pi} \delta \int_{\partial \Sigma} k^{\gamma} A_{\gamma} F^{\alpha \beta} d S_{\alpha \beta} \\
& +\int_{\Sigma} \Delta\left[A_{\beta}\left(j^{\beta} k^{\alpha}-j^{\alpha} k^{\beta}\right) d S_{\alpha}\right] \\
& -\frac{1}{8 \pi} \delta \int_{\Sigma}\left[G_{\beta}^{\alpha}-8 \pi\left(T_{\beta}^{\alpha}+T_{\mathrm{F} \beta}^{\alpha}\right)\right] k^{\beta} d S_{\alpha}, \\
& -\frac{1}{4 \pi} \delta \int_{\Sigma} k^{\gamma} A_{\gamma}\left(\nabla_{\beta} F^{\alpha \beta}-4 \pi j^{\alpha}\right) d S_{\alpha} .
\end{aligned}
$$

The integrand of the second term becomes

$$
\begin{aligned}
& \Delta\left[(\epsilon+p) u^{\alpha} u_{\beta} v^{\beta} d S_{\alpha}\right] \\
= & h u_{\beta} v^{\beta} \Delta\left(\rho u^{\alpha} d S_{\alpha}\right)+v^{\beta} \Delta\left(h u_{\beta}\right) \rho u^{\alpha} d S_{\alpha} \\
& +(\epsilon+p) u^{\alpha} u^{\beta} £_{k} \xi^{\beta} d S_{\alpha},
\end{aligned}
$$

where $\Delta v^{\beta}=-\Delta k^{\beta}=£_{k} \xi^{\beta}$ was used, and the integrand of the fifth term is

$$
\begin{aligned}
& \Delta\left[A_{\beta}\left(j^{\beta} k^{\alpha}-j^{\alpha} k^{\beta}\right) d S_{\alpha}\right] \\
&= \Delta A_{\beta}\left(j^{\beta} k^{\alpha}-j^{\alpha} k^{\beta}\right) d S_{\alpha} \\
&+A_{\beta} \frac{1}{\sqrt{-g}} \Delta\left(j^{\beta} \sqrt{-g}\right) k^{\alpha} d S_{\alpha} \\
&-A_{\beta} k^{\beta} \Delta\left(j^{\alpha} d S_{\alpha}\right) \\
&+A_{\beta}\left(j^{\alpha} £_{k} \xi^{\beta}-j^{\beta} £_{k} \xi^{\alpha}\right) d S_{\alpha}
\end{aligned}
$$

where $\Delta k^{\alpha}=£_{\xi} k^{\alpha}=-£_{k} \xi^{\alpha}$, because of our gauge choice $\delta k^{\alpha}=0$.

The variation of $Q_{L}-\sum_{i} Q_{L i}$ is given by

$$
\begin{aligned}
\delta & \left(Q_{L}-\sum_{i} Q_{L i}\right) \\
& =\oint_{\partial \Sigma}\left(k^{\alpha} \Theta^{\beta}-k^{\beta} \Theta^{\alpha}\right) d S_{\alpha \beta} \\
& =\int_{\Sigma} \nabla_{\beta}\left(k^{\alpha} \Theta^{\beta}-k^{\beta} \Theta^{\alpha}\right) d S_{\alpha} \\
& =\int_{\Sigma} \nabla_{\beta} \Theta^{\beta} k^{\alpha} d S_{\alpha}-\int_{\Sigma} £_{k} \Theta^{\alpha} d S_{\alpha},
\end{aligned}
$$

where we have used the relation $\nabla_{\alpha} k^{\alpha}=0$ to obtain the last equality. The integrand of the last term in Eq. (B12) is written as

$$
\begin{aligned}
£_{k} \Theta^{\alpha} d S_{\alpha}= & (\epsilon+p) q^{\alpha} £_{k} \xi^{\beta} d S_{\alpha} \\
& +A_{\beta}\left(j^{\alpha} £_{k} \xi^{\beta}-j^{\beta} £_{k} \xi^{\alpha}\right) d S_{\alpha} \\
= & (\epsilon+p) u^{\alpha} u_{\beta} £_{k} \xi^{\beta} d S_{\alpha} \\
& +A_{\beta}\left(j^{\alpha} £_{k} \xi^{\beta}-j^{\beta} £_{k} \xi^{\alpha}\right) d S_{\alpha},
\end{aligned}
$$

where we used the fact that $\xi^{\alpha}$ as well as its Lie derivative along $k^{\alpha}$ is spatial $£_{k} \xi^{\alpha} \nabla_{\alpha} t=0$. These two terms 
in Eq. (B13) cancel out with the last terms of Eqs. (B10) and (B11). Note that the current $j^{\alpha}$ respects the sym- metry $£_{k} j^{\alpha}=0$.

Finally, we obtain an expression for $\delta\left(Q-\sum_{i} Q_{i}\right)$ :

$$
\begin{aligned}
\delta(Q- & \left.\sum_{i} Q_{i}\right) \\
= & \int_{\Sigma}\left\{\frac{T}{u^{t}} \Delta s \rho u^{\alpha} d S_{\alpha}+\left[\frac{h}{u^{t}}+h u_{\beta} v^{\beta}\right] \Delta\left(\rho u^{\alpha} d S_{\alpha}\right)+v^{\beta} \Delta\left(h u_{\beta}\right) \rho u^{\alpha} d S_{\alpha}\right. \\
& \left.-A_{\beta} k^{\beta} \Delta\left(j^{\alpha} d S_{\alpha}\right)-\left(j^{\alpha} k^{\beta}-j^{\beta} k^{\alpha}\right) \Delta A_{\beta} d S_{\alpha}\right\}-\frac{1}{4 \pi} \delta \int_{\Sigma} F^{\alpha \gamma} £_{k} A_{\gamma} d S_{\alpha}+\frac{1}{4 \pi} \delta \int_{\partial \Sigma} k^{\gamma} A_{\gamma} F^{\alpha \beta} d S_{\alpha \beta} \\
- & \frac{1}{8 \pi} \delta \int_{\Sigma}\left[G^{\alpha}{ }_{\beta}-8 \pi\left(T^{\alpha}{ }_{\beta}+T_{\mathrm{F} \beta}^{\alpha}\right)\right] k^{\beta} d S_{\alpha}-\frac{1}{4 \pi} \delta \int_{\Sigma} k^{\gamma} A_{\gamma}\left(\nabla_{\beta} F^{\alpha \beta}-4 \pi j^{\alpha}\right) d S_{\alpha} \\
+ & \int_{\Sigma}\left\{\frac{1}{16 \pi}\left[G^{\alpha \beta}-8 \pi\left(T^{\alpha \beta}+T_{\mathrm{F}}^{\alpha \beta}\right)\right] \delta g_{\alpha \beta}+\frac{1}{4 \pi}\left(\nabla_{\beta} F^{\alpha \beta}-4 \pi j^{\alpha}\right) \delta A_{\alpha}+\xi^{\alpha}\left[\nabla_{\beta} T_{\alpha}{ }^{\beta}-F_{\alpha \beta} j^{\beta}\right]\right\} k^{\gamma} d S_{\gamma} .
\end{aligned}
$$

Note that $k^{\alpha} d S_{\alpha}=\sqrt{-g} d^{3} x$. When the field equations, their perturbations, and equations of motion are satisfied, using $£_{k} A_{\alpha}=0$, and Eq. (9) noting $\int_{\partial \Sigma}=\oint_{S}-\sum_{i} \oint_{\mathcal{B} i}$, Eq. (B14) is rewritten

$$
\begin{aligned}
\delta\left(Q-\sum_{i} Q_{i}\right)= & \int_{\Sigma}\left\{\frac{T}{u^{t}} \Delta s \rho u^{\alpha} d S_{\alpha}+\left[\frac{h}{u^{t}}+h u_{\beta} v^{\beta}\right] \Delta\left(\rho u^{\alpha} d S_{\alpha}\right)+v^{\beta} \Delta\left(h u_{\beta}\right) \rho u^{\alpha} d S_{\alpha}\right. \\
& \left.-A_{\beta} k^{\beta} \Delta\left(j^{\alpha} d S_{\alpha}\right)-\left(j^{\alpha} k^{\beta}-j^{\beta} k^{\alpha}\right) \Delta A_{\beta} d S_{\alpha}\right\}-\sum_{i} \frac{1}{4 \pi} \delta \oint_{\mathcal{B} i} k^{\gamma} A_{\gamma} F^{\alpha \beta} d S_{\alpha \beta} .
\end{aligned}
$$

\section{Appendix C: Calculation of Eq. (74)}

A relation used in Eq. (74) is proved in 30], which is repeated here for a reference. Consider a closed 2form $F_{\alpha \beta}\left((d F)_{\alpha \beta \gamma}=0\right)$, and a vector $N^{\alpha}$ such that $F_{\alpha \beta} N^{\beta}=0$. Then, for any vector $q^{\alpha}$, a relation

$$
(d \eta)_{\alpha \beta} N^{\beta}=F_{\alpha \beta} £_{q} N^{\beta}
$$

is satisfied, where $\eta_{\alpha}$ is defined by $\eta_{\alpha}=F_{\alpha \beta} q^{\beta}$. This can be shown as follows:

$$
\begin{aligned}
(d \eta)_{\alpha \beta} N^{\beta} & =(d(F \cdot q))_{\alpha \beta} N^{\beta} \\
& =\left[(q \cdot d F)_{\alpha \beta}-£_{q} F_{\alpha \beta}\right] N^{\beta} \\
& =F_{\alpha \beta} £_{q} N^{\beta} .
\end{aligned}
$$

The Cartan identity was used in the second equality and the relation $F_{\alpha \beta} N^{\beta}=0$ in third one.

\section{Appendix D: First integral of MHD-Euler equation in BGSM formulation}

A formulation for uniformly rotating axisymmetric stars with poloidal magnetic fields is derived in [37]. In this section, we show that the Bekenstein and Oron formulation of ideal MHD includes a first integral of the MHD-Euler equation derived in the BGSM formulation, assuming the same symmetry and suitably choosing an auxiliary vector $q^{\alpha}$ in the current (71).
In the BGSM formulation, a stationary, axisymmetric and circular spacetime is assumed. And more specifically the flow field of rotating star is assumed to be uniform; with a constant angular velocity $\Omega, 4$-velocity is written $u^{\alpha}=u^{t} k^{\alpha}=u^{t}\left(t^{\alpha}+\Omega \phi^{\alpha}\right)$ where $t^{\alpha}$ and $\phi^{\alpha}$ are killing vectors.

Carter has shown 22] that in stationary, axisymmetric and circular spacetime, the vector potential and the current are such that $A_{\alpha}=A_{t} \nabla_{\alpha} t+A_{\phi} \nabla_{\alpha} \phi$ and $j^{\alpha}=$ $j^{t} t^{\alpha}+j^{\phi} \phi^{\alpha}$ respectively. Since the vector potential $A_{\alpha}$ is assumed to respect the symmetry $£_{k} A_{\alpha}=0$, the ideal MHD condition $F_{\alpha \beta} u^{\beta}=0$ implies, for a corotating flow,

$$
F_{\alpha \beta} k^{\beta}=-£_{k} A_{\alpha}+\nabla_{\alpha}\left(A_{\beta} k^{\beta}\right)=\nabla_{\alpha}\left(A_{\beta} k^{\beta}\right)=0,
$$

hence

$$
A_{\alpha} k^{\alpha}=A_{t}+\Omega A_{\phi}=\text { constant. }
$$

Using this relation, the vector potential is written

$$
A_{\alpha}=A_{\phi}\left(\nabla_{\alpha} \phi-\Omega \nabla_{\alpha} t\right) .
$$

Note that $\nabla_{\alpha} \phi-\Omega \nabla_{\alpha} t$ is orthogonal to the helical vector, $k^{\alpha}\left(\nabla_{\alpha} \phi-\Omega \nabla_{\alpha} t\right)=0$.

Rewriting the current as

$$
j^{\alpha}=j^{t} k^{\alpha}+J \phi^{\alpha},
$$

with $J=j^{\alpha}\left(\nabla_{\alpha} \phi-\Omega \nabla_{\alpha} t\right)=j^{\phi}-\Omega j^{t}$, the Lorenz force becomes

$$
\frac{1}{\rho} F_{\alpha \beta} j^{\beta}=\frac{J}{\rho} F_{\alpha \beta} \phi^{\beta}=\frac{J}{\rho}\left[-£_{\phi} A_{\alpha}+\nabla_{\alpha}\left(A_{\beta} \phi^{\beta}\right)\right] .
$$


Then, with the symmetry $£_{\phi} A_{\alpha}=0$, the MHD-Euler equation (70) is written

$$
k^{\beta}(d(h \underline{\mathrm{u}}))_{\beta \alpha}=\frac{J}{\rho u^{t}} \nabla_{\alpha}\left(A_{\beta} \phi^{\beta}\right),
$$

or using $k^{\beta}(d(h \underline{\mathrm{u}}))_{\beta \alpha}=-\nabla_{\alpha}\left(h u_{\beta} k^{\beta}\right)=\nabla_{\alpha}\left(h / u^{t}\right)$,

$$
\nabla_{\alpha}\left(\frac{h}{u^{t}}\right)-\frac{J}{\rho u^{t}} \nabla_{\alpha} A_{\phi}=0 .
$$

Hence, an integrability condition of this equation is

$$
\frac{J}{\rho u^{t}}=f\left(A_{\phi}\right) \text {. }
$$

Equation (D7) and MHD-Euler equation for the comoving flow (109) with the current (71) agree if the relation

$$
F_{\alpha \beta} £_{k} q^{\beta}=-\frac{J}{\rho u^{t}} \nabla_{\alpha} A_{\phi}
$$

is satisfied. For example, if the vector $q^{\alpha}$ satisfies

$$
£_{k} q^{\alpha}=-\frac{J}{\rho u^{t}} \phi^{\alpha}=f\left(A_{\phi}\right) \phi^{\alpha},
$$

the Bekenstein and Oron formulation becomes the BGSM formulation [cf. Eq. (D5)].

\section{Appendix E: Calculation of $\delta\left(Q-\sum_{i} Q_{i}\right)$ for the}

\section{Lagrangian with Bekenstein and Oron's interaction} term

In the Bekenstein and Oron theory, the ideal MHD condition $F_{\alpha \beta} u^{\beta}=0$ is imposed by adding a constraint to the Lagrangian density with a Lagrange multiplier $q^{\alpha}$,

$$
\mathcal{L}_{\mathrm{I}}=F_{\alpha \beta} \rho u^{\alpha} q^{\beta} \sqrt{-g} .
$$

This term replaces an interaction term, $A_{\alpha} j^{\alpha} \sqrt{-g}$, of the field and electric current. The variation of $\mathcal{L}_{\mathrm{I}}$ becomes,

$$
\begin{aligned}
& \frac{1}{\sqrt{-g}} \delta \mathcal{L}_{\mathrm{I}}=-\nabla_{\beta} \delta A_{\alpha}\left(\rho u^{\alpha} q^{\beta}-\rho u^{\beta} q^{\alpha}\right) \\
& +\frac{1}{\sqrt{-g}} F_{\alpha \beta}\left[\Delta\left(\rho u^{\alpha} q^{\beta} \sqrt{-g}\right)-£_{\xi}\left(\rho u^{\alpha} q^{\beta} \sqrt{-g}\right)\right],
\end{aligned}
$$

The last term is calculated as

$$
\begin{aligned}
& -\frac{1}{\sqrt{-g}} F_{\alpha \beta} £_{\xi}\left(\rho u^{\alpha} q^{\beta} \sqrt{-g}\right) \\
& =\rho u^{\alpha} q^{\beta}\left[\xi^{\gamma}(d F)_{\gamma \alpha \beta}+d(\xi \cdot F)_{\alpha \beta}\right] \\
& +\nabla_{\alpha}\left(F_{\beta \gamma} \rho u^{\gamma} q^{\beta} \xi^{\alpha}\right),
\end{aligned}
$$

where the Cartan identity for the 2-form $F_{\alpha \beta}, £_{\xi} F_{\alpha \beta}=$ $\xi^{\gamma}(d F)_{\gamma \alpha \beta}+(d(\xi \cdot F))_{\alpha \beta}$ is used, and

$$
\begin{aligned}
& \rho u^{\alpha} q^{\beta}(d(\xi \cdot F))_{\alpha \beta}=\left(\rho u^{\alpha} q^{\beta}-\rho u^{\beta} q^{\alpha}\right) \nabla_{\alpha}\left(\xi^{\gamma} F_{\gamma \beta}\right) \\
& =\xi^{\alpha} F_{\alpha \beta} j^{\beta}+\nabla_{\alpha}\left[\left(\rho u^{\alpha} q^{\beta}-\rho u^{\beta} q^{\alpha}\right) \xi^{\gamma} F_{\gamma \beta}\right] .
\end{aligned}
$$

Hence, using $j^{\alpha}=\nabla_{\beta}\left(\rho u^{\alpha} q^{\beta}-\rho u^{\beta} q^{\alpha}\right)$, we have

$$
\begin{aligned}
& \frac{1}{\sqrt{-g}} \delta \mathcal{L}_{\mathrm{I}}=\frac{1}{\sqrt{-g}} F_{\alpha \beta} \Delta\left(\rho u^{\alpha} q^{\beta} \sqrt{-g}\right) \\
& +j^{\alpha} \delta A_{\alpha}+\xi^{\alpha}\left[F_{\alpha \beta} j^{\beta}+\rho u^{\beta} q^{\gamma}(d F)_{\alpha \beta \gamma}\right]+\nabla_{\alpha} \Theta_{\mathrm{I}}^{\alpha},
\end{aligned}
$$

where

$$
\begin{aligned}
\Theta_{\mathrm{I}}^{\alpha} & =\left(\rho u^{\alpha} q^{\beta}-\rho u^{\beta} q^{\alpha}\right) \delta A_{\beta} \\
& -\left(\rho u^{\alpha} q^{\beta} \xi^{\gamma}+\rho u^{\beta} q^{\gamma} \xi^{\alpha}+\rho u^{\gamma} q^{\alpha} \xi^{\beta}\right) F_{\beta \gamma} .
\end{aligned}
$$

To calculate the difference of Noether charge $\delta(Q-$ $\left.\sum_{i} Q_{i}\right)$, we first associate $Q_{K}-\sum_{i} Q_{K i}$ with the Lagrangian (152) as

$$
\begin{aligned}
& Q_{K}-\sum_{i} Q_{K i} \\
= & -\int_{\Sigma} \mathcal{L} d^{3} x+\int_{\Sigma}(\epsilon+p) u^{\alpha} u_{\beta} v^{\beta} d S_{\alpha} \\
& -\frac{1}{4 \pi} \int_{\Sigma} F^{\alpha \gamma} £_{k} A_{\gamma} d S_{\alpha}+\frac{1}{4 \pi} \int_{\partial \Sigma} k^{\gamma} A_{\gamma} F^{\alpha \beta} d S_{\alpha \beta} \\
& +\int_{\Sigma} F_{\alpha \beta} \rho u^{\alpha} q^{\beta} k^{\gamma} d S_{\gamma}-\int_{\Sigma} k^{\gamma} A_{\gamma} j^{\alpha} d S_{\alpha} . \\
& -\frac{1}{8 \pi} \int_{\Sigma}\left[G^{\alpha}{ }_{\beta}-8 \pi\left(T_{\beta}^{\alpha}+T_{\mathrm{F} \beta}^{\alpha}\right)\right] k^{\beta} d S_{\alpha} \\
& -\frac{1}{4 \pi} \int_{\Sigma} k^{\gamma} A_{\gamma}\left(\nabla_{\beta} F^{\alpha \beta}-4 \pi j^{\alpha}\right) d S_{\alpha},
\end{aligned}
$$

which corresponds to Eq. (B8). The integrand of the fifth term in the r.h.s. of Eq. (E7) is rewritten

$$
\begin{aligned}
& F_{\alpha \beta} \rho u^{\alpha} q^{\beta} k^{\gamma} d S_{\gamma}=F_{\alpha \beta} \rho\left(k^{\alpha}+v^{\alpha}\right) q^{\beta} u^{\gamma} d S_{\gamma} \\
= & k^{\alpha} F_{\alpha \beta} q^{\beta} \rho u^{\gamma} d S_{\gamma}+v^{\alpha} \eta_{\alpha} \rho u^{\gamma} d S_{\gamma},
\end{aligned}
$$

and combined with the sixth term as

$$
\begin{aligned}
& v^{\alpha} \eta_{\alpha} \rho u^{\beta} d S_{\beta}-\nabla_{\alpha}\left(k^{\beta} A_{\beta}\right) q^{\alpha} \rho u^{\gamma} d S_{\gamma}-k^{\beta} A_{\beta} j^{\alpha} d S_{\alpha} \\
& =v^{\alpha} \eta_{\alpha} \rho u^{\beta} d S_{\beta}-£_{q}\left(k^{\beta} A_{\beta} \rho u^{\gamma} d S_{\gamma}\right)
\end{aligned}
$$

where Eq. (98) and $£_{k} A_{\alpha}=0$ were used. The integral of the last term of Eq. (E9) over $\Sigma$ is rewritten a surface integral over $\partial \Sigma$ that vanishes, because of the gauge invariance under the transformation $q^{\alpha} \rightarrow q^{\alpha}+\lambda u^{\alpha}$ which can always be used to make $q^{\alpha}$ spatial, $q^{\alpha} \nabla_{\alpha} t=0$.

The third line of Eq. (E7) is replaced by $v^{\alpha} \eta_{\alpha} \rho u^{\beta} d S_{\beta}$, then a variation of the charge is calculated.

A difference from the calculation of $\delta\left(Q_{K}-\sum_{i} Q_{K i}\right)$ in Appendix $\mathrm{B}$ is the terms,

$$
\begin{aligned}
& \Delta\left[(\epsilon+p) u^{\alpha} u_{\beta} v^{\beta} d S_{\alpha}\right]+\Delta\left(v^{\beta} \eta_{\beta} \rho u^{\alpha} d S_{\alpha}\right) \\
= & \left(h u_{\beta}+\eta_{\beta}\right) v^{\beta} \Delta\left(\rho u^{\alpha} d S_{\alpha}\right)+v^{\beta} \Delta\left(h u_{\beta}+\eta_{\beta}\right) \rho u^{\alpha} d S_{\alpha} \\
+ & (\epsilon+p) u^{\alpha} u_{\beta} £_{k} \xi^{\beta} d S_{\alpha}+£_{k} \xi^{\beta} \eta_{\beta} \rho u^{\alpha} d S_{\alpha}, \quad(\mathrm{E} 10)
\end{aligned}
$$


where $\Delta v^{\beta}=-\Delta k^{\beta}=£_{k} \xi^{\beta}$ is used. In the calculation of $\delta\left(Q_{L}-\sum_{i} Q_{L i}\right)$, a term $£_{k} \Theta^{\alpha} d S_{\alpha}$ becomes,

$$
\begin{aligned}
£_{k} \Theta^{\alpha} d S_{\alpha} & =(\epsilon+p) u^{\alpha} u_{\beta} £_{k} \xi^{\beta} d S_{\alpha} \\
& +£_{k} \xi^{\gamma} F_{\gamma \beta}\left(\rho u^{\alpha} q^{\beta}-\rho u^{\beta} q^{\alpha}\right) d S_{\alpha} \\
& +\left(\delta A_{\beta}+\xi^{\gamma} F_{\gamma \beta}\right)\left(\rho u^{\alpha} £_{k} q^{\beta}-\rho u^{\beta} £_{k} q^{\alpha}\right) d S_{\alpha}
\end{aligned}
$$

where $\xi^{\alpha}$ and $£_{k} \xi^{\alpha}$ are both spatial. The first term and a part of the second term in the r.h.s. of Eq. (E11) cancel out with the last two terms in Eq. (E10). With the Cartan identity, $\xi^{\gamma} F_{\gamma \beta}=£_{\xi} A_{\beta}-\nabla_{\beta}\left(\xi^{\gamma} A_{\gamma}\right)$ the last term of
Eq. (E11) becomes

$$
\begin{aligned}
& \left(\delta A_{\beta}+\xi^{\gamma} F_{\gamma \beta}\right)\left(\rho u^{\alpha} £_{k} q^{\beta}-\rho u^{\beta} £_{k} q^{\alpha}\right) d S_{\alpha} \\
= & {\left[\Delta A_{\beta}-\nabla_{\beta}\left(\xi^{\gamma} A_{\gamma}\right)\right]\left(\rho u^{\alpha} £_{k} q^{\beta}-\rho u^{\beta} £_{k} q^{\alpha}\right) d S_{\alpha} } \\
= & \Delta A_{\beta}\left(\rho u^{\alpha} £_{k} q^{\beta}-\rho u^{\beta} £_{k} q^{\alpha}\right) d S_{\alpha}+\xi^{\gamma} A_{\gamma} £_{k} j^{\alpha} d S_{\alpha} \\
- & \nabla_{\beta}\left[\xi^{\gamma} A_{\gamma}\left(\rho u^{\alpha} £_{k} q^{\beta}-\rho u^{\beta} £_{k} q^{\alpha}\right)\right] d S_{\alpha}, \quad(\mathrm{E} 12
\end{aligned}
$$

where the second term of the r.h.s. of the last equality vanishes for the symmetry, $£_{k} j^{\alpha}=0$, and an integral of the last term over $\Sigma$ vanishes for the Stokes theorem.

Finally, we obtain an expression for $\delta\left(Q-\sum_{i} Q_{i}\right)$ for the Bekenstein and Oron ideal MHD theory:

$$
\begin{aligned}
\delta & \left(Q-\sum_{i} Q_{i}\right) \\
= & \int_{\Sigma}\left\{\frac{T}{u^{t}} \Delta s \rho u^{\alpha} d S_{\alpha}+\left[\frac{h}{u^{t}}+\left(h u_{\beta}+\eta_{\beta}\right) v^{\beta}\right] \Delta\left(\rho u^{\alpha} d S_{\alpha}\right)+v^{\beta} \Delta\left(h u_{\beta}+\eta_{\beta}\right) \rho u^{\alpha} d S_{\alpha}\right. \\
& \left.-\left(\rho u^{\alpha} £_{k} q^{\beta}-\rho u^{\beta} £_{k} q^{\alpha}\right) \Delta A_{\beta} d S_{\alpha}\right\}+\frac{1}{4 \pi} \delta \oint_{\partial \Sigma} k^{\gamma} A_{\gamma} F^{\alpha \beta} d S_{\alpha \beta}+\int_{\Sigma} F_{\alpha \beta} u^{\beta}\left[\frac{1}{u^{t}} \Delta\left(q^{\alpha} \rho u^{\gamma} d S_{\gamma}\right)+£_{k} \xi^{\alpha} \rho q^{\gamma} d S_{\gamma}\right] \\
- & \frac{1}{8 \pi} \delta \int_{\Sigma}\left[G^{\alpha}{ }_{\beta}-8 \pi\left(T_{\beta}^{\alpha}+T_{\mathrm{F} \beta}^{\alpha}\right)\right] k^{\beta} d S_{\alpha}-\frac{1}{4 \pi} \delta \int_{\Sigma} k^{\gamma} A_{\gamma}\left(\nabla_{\beta} F^{\alpha \beta}-4 \pi j^{\alpha}\right) d S_{\alpha} \\
+ & \int_{\Sigma}\left\{\frac{1}{16 \pi}\left[G^{\alpha \beta}-8 \pi\left(T^{\alpha \beta}+T_{\mathrm{F}}^{\alpha \beta}\right)\right] \delta g_{\alpha \beta}+\frac{1}{4 \pi}\left(\nabla_{\beta} F^{\alpha \beta}-4 \pi j^{\alpha}\right) \delta A_{\alpha}\right. \\
& \left.+\xi^{\alpha}\left[\nabla_{\beta} T_{\alpha}{ }^{\beta}-F_{\alpha \beta} j^{\beta}-\rho u^{\beta} q^{\gamma}(d F)_{\alpha \beta \gamma}\right]\right\} k^{\delta} d S_{\delta} .
\end{aligned}
$$

This expression is compared with Eq. (B14). Note that, in the second line of Eq. (E13), the circulation of mag- netized flow explicitly appears as in Eq. (84).
[1] P. M. Woods, \& C. Thompson, In "Compact stellar X-ray sources" edited by W. Lewin \& M. van der Klis, Cambridge Astrophysics Series, No. 39, 547, Cambridge University Press (2006).

[2] J. K. Blackburn and S. Detweiler, Phys. Rev. D 46, 2318 (1992); S. Detweiler, Phys. Rev. D 50, 4929 (1994).

[3] J. L. Friedman and K. Uryu, Phys. Rev. D 73, 104039 (2006); M. M. Glenz and K. Uryu, Phys. Rev. D 76, 027501 (2007).

[4] A. Schild, Phys. Rev. 131, 2762 (1963).

[5] S. Bonazzola, E. Gourgoulhon and J.-A. Marck, Phys. Rev. D 56, 7740 (1997).

[6] H. Asada, Phys. Rev. D 57, 7292 (1998).

[7] J. L. Friedman, K. Uryu and M. Shibata (FUS), Phys. Rev. D 65, 064035 (2002) [Erratum-ibid. D 70, 129904 (2004)]

[8] E. Gourgoulhon, P. Grandclement and S. Bonazzola, Phys. Rev. D 65, 044020 (2002); P. Grandclement, E. Gourgoulhon and S. Bonazzola, Phys. Rev. D 65,
044021 (2002).

[9] C. Klein, Phys. Rev. D 70, 124026 (2004)

[10] C. G. Torre, J. Math. Phys. 44, 6223 (2003); C. G. Torre, J. Math. Phys. 47, 073501 (2006)

[11] J. P. Bruneton, AIP Conf. Proc. 861, 558 (2006).

[12] R. Beig, J. M. Heinzle and B. G. Schmidt, Phys. Rev. Lett. 98, 121102 (2007); J. Bicak and B. G. Schmidt, Phys. Rev. D 76, 104040 (2007).

[13] J. T. Whelan, C. Beetle, W. Landry and R. H. Price, Class. Quant. Grav. 19, 1285 (2002); Z. Andrade et al., Phys. Rev. D 70, 064001 (2004); S. R. Lau and R. H. Price, J. Comput. Phys. 227, 1126 (2007); C. Beetle, B. Bromley, N. Hernandez and R. H. Price, Phys. Rev. D 76, 084016 (2007); N. Hernandez and R. H. Price, Phys. Rev. D 79, 064008 (2009).

[14] S. Yoshida, B. C. Bromley, J. S. Read, K. Uryu and J. L. Friedman, Class. Quant. Grav. 23, S599 (2006).

[15] J. Lee and R. M. Wald, J. Math. Phys. 31, 725 (1990).

[16] R. M. Wald, Phys. Rev. D. 48, R3427 (1993). 
[17] V. Iyer and R. M. Wald, Phys. Rev. D. 52, 4430 (1995).

[18] V. Iyer, Phys. Rev. D. 55, 3411 (1997).

[19] B. F. Schutz and R.D. Sorkin, Annals of Phys., 107,1 (1977).

[20] R.D. Sorkin, Proceedings of the Royal Society London A 435: 635-644 (1991).

[21] J. D. Brown, Class. Quant. Grav. 10, 1579 (1993).

[22] B. Carter, in Black holes - Les Houches 1972, edited by C. DeWitt \& B.S. DeWitt, Gordon and Breach, New York (1973), p. 57.

[23] B. Carter, in General Relativity: An Einstein centenary survey, edited by S. W. Hawking and W. Israel (1979), p. 294; B. Carter, in Anneau d'accrétion sur les trous noirs, edited by I. Moret-Bailly and C. Latremolière, Faculté des Sciences (Angers) (1979), p. 166; B. Carter, in Active Galactic Nuclei, edited by C. Hazard and S. Mitton, Cambridge University Press (Cambridge) (1979), p. 273.

[24] S. Bonazzola, E. Gourgoulhon, and J.-A. Marck, Phys. Rev. Lett. 82, 892 (1999); E. Gourgoulhon, P. Grandclement, K. Taniguchi, J.-A. Marck, S. Bonazzola, Phys. Rev. D 63, 064029 (2001); K. Taniguchi and E. Gourgoulhon, Phys. Rev. D 66, 104019 (2002); ibid. 68, 124025 (2003); M. Bejger, D. Gondek-Rosinska, E. Gourgoulhon, P. Haensel, K. Taniguchi and J. L. Zdunik, Astron. Astrophys. 431, 297 (2005). K. Taniguchi and M. Shibata, Astrophys. J. Suppl. 188, 187 (2010)

[25] K. Uryu and Y. Eriguchi, Phys. Rev. D 61, 124023 (2000); K. Uryu, M. Shibata, and Y. Eriguchi, Phys. Rev. D 62, 104015 (2000); K. Uryu, F. Limousin, J. L. Friedman, E. Gourgoulhon and M. Shibata, Phys. Rev. Lett. 97, 171101 (2006); K. Uryu, F. Limousin, J. L. Friedman, E. Gourgoulhon and M. Shibata, Phys. Rev. D 80, 124004 (2009).

[26] K. Taniguchi, T. W. Baumgarte, J. A. Faber and S. L. Shapiro, Phys. Rev. D 72, 044008 (2005); K. Taniguchi, T. W. Baumgarte, J. A. Faber and S. L. Shapiro, Phys. Rev. D 74, 041502(R) (2006); K. Taniguchi, T. W. Baumgarte, J. A. Faber and S. L. Shapiro, Phys. Rev. D 75, 084005 (2007); K. Taniguchi, T. W. Baumgarte, J. A. Faber and S. L. Shapiro, Phys. Rev. D 77, 044003 (2008); K. Kyutoku, M. Shibata, and K. Taniguchi, Phys. Rev. D 79, 124018 (2009); F. Foucart, L. E. Kidder, H. P. Pfeiffer and S. A. Teukolsky, Phys. Rev. D 77, 124051 (2008).

[27] G. B. Cook, Living Rev. Rel. 3, 5 (2000); G. B. Cook, Phys. Rev. D 50, 5025 (1994); S. Brandt and B. Brügmann, Phys. Rev. Lett. 78, 3606 (1997); H. P. Pfeiffer, S. A. Teukolsky and G. B. Cook, Phys. Rev. D 62, 104018 (2000); P. Marronetti and R. A. Matzner, Phys. Rev. Lett. 85, 5500 (2000); G. B. Cook, Phys. Rev. D 65, 084003 (2002); H. P. Pfeiffer, G. B. Cook and S. A. Teukolsky, Phys. Rev. D 66,
024047 (2002); G. Lovelace, R. Owen, H. P. Pfeiffer and T. Chu, Phys. Rev. D 78, 084017 (2008); M. Caudill, G. B. Cook, J. D. Grigsby and H. P. Pfeiffer, Phys. Rev. D 74, 064011 (2006); W. Tichy, B. Brügmann, M. Campanelli and P. Diener, Phys. Rev. D 67, 064008 (2003); B. J. Kelly, W. Tichy, M. Campanelli and B. F. Whiting, Phys. Rev. D 76, 024008 (2007).

[28] J. D. Bekenstein and E. Oron, Phys. Rev. D , 18, 1809 (1978); J. D. Bekenstein, Astrophys. J. , 319, 207 (1987).

[29] J. D. Bekenstein and A. Oron, Phys. Rev. E 62, 5594 (2000); J. D. Bekenstein and A. Oron, Found. Phys. 31, 895 (2001);

[30] J. D. Bekenstein and G. Betschart, Phys. Rev. D 74, 083009 (2006).

[31] I. E. Tarapov, PMM U.S.S.R., 48, 275 (1984); V. B. Gorskii, PMM U.S.S.R., 50, 388 (1986).

[32] C. S. Kochanek, Astrophys. J. 398, 234 (1992): L. Bildsten and C. Cutler, Astrophys. J. 400, 175 (1992).

[33] H. Friedrich, I. Rácz, and R. M. Wald, Comm. Math. Phys. 204, 691 (1999).

[34] J. M. Bardeen, B. Carter, and S. W. Hawking, Comm. Math. Phys. 31, 161 (1973).

[35] E. Gourgoulhon, in Stellar Fluid Dynamics and Numerical Simulations: From the Sun to Neutron Stars, edited by M. Rieutord \& B. Dubrulle, EAS Publications Series 21, 43 (2006).

[36] S. Bonazzola, E. Gourgoulhon and J.-A. Marck, Phys. Rev. D 56, 7740 (1997).

[37] S. Bonazzola, E. Gourgoulhon, M. Salgado and J.-A. Marck, Astron. Astrophys. 278, 421 (1993).

[38] J. L. Friedman, Comm. Math. Phys. 62, 247 (1978).

[39] B. Carter, Comm. Math. Phys. 30, 261 (1973).

[40] A. H. Taub, Comm. Math. Phys. 15, 235 (1969).

[41] K. Uryu and Y. Eriguchi, Mon. Not. Roy. Astron. Soc. 296, L1 (1998); K. Uryu and Y. Eriguchi, Mon. Not. Roy. Astron. Soc. 299, 575 (1998); K. Uryu and Y. Eriguchi, Astrophys. J. Suppl. 118, 563 (1998); Y. Eriguchi and K. Uryu, Prog. Theor. Phys. Suppl. 136, 199 (1999); K. Uryu and Y. Eriguchi, Mon. Not. Roy. Astron. Soc. 303, 329 (1999); K. Taniguchi, Prog. Theor. Phys. 101, 283 (1999); K. Taniguchi and T. Nakamura, Phys. Rev. Lett. 84, 581 (2000); K. Taniguchi and T. Nakamura, Phys. Rev. D 62, 044040 (2000); K. Taniguchi, E. Gourgoulhon and S. Bonazzola, Phys. Rev. D 64, 064012 (2001); K. Taniguchi and E. Gourgoulhon, Phys. Rev. D 65, 044027 (2002).

[42] H. Asada, Phys. Rev. D 57, 7292 (1998); M. Shibata, Phys. Rev. D 58, 024012 (1998); S. A. Teukolsky, Astrophys. J. , 504, 442 (1998)

[43] B. F. Schutz, Phys. Rev. D 2, 2762 (1970).

[44] R. Sorkin, Astrophys. J. 249, 254 (1981). 